Supporting Information for

\title{
Dimethylsilyl Ketene Acetal as a Nucleophile in Asymmetric Michael Reaction: Enhanced Enantioselectivity in Oxazaborolidinone-Catalyzed Reaction
}

\author{
Toshiro Harada, * Shinya Adachi, and Xiaowei Wang \\ Department of Chemistry and Materials Technology, Kyoto Institute of Technology, Matsugasaki, \\ Sakyo-ku, Kyoto 606-8585, Japan \\ harada@chem.kit.ac.jp
}

1-(tert-Butylthio)-1-dimethylsilyloxyethene (6b): To a solution of diisopropylamine (16 $\mathrm{mL}, 114 \mathrm{mmol})$ in THF $(175 \mathrm{~mL})$ was added BuLi $(1.6 \mathrm{M}$ in hexane, $71 \mathrm{~mL}, 114 \mathrm{mmol})$ over 5 $\min$ at $0{ }^{\circ} \mathrm{C}$. After being stirred for $10 \mathrm{~min}$, the mixture was cooled to $-78{ }^{\circ} \mathrm{C}$. S-tert-butyl thioacetate $(14 \mathrm{~mL}, 95 \mathrm{mmol})$ was added to the stirred solution of thus prepared LDA over 60 min. After $1.5 \mathrm{~h}$, chlorodimethylsilane $(13 \mathrm{~mL}, 114 \mathrm{mmol})$ was added to the reaction mixture over $30 \mathrm{~min}$ at $-78^{\circ} \mathrm{C}$. The mixture was allowed to warm to room temperature overnight. The resulting mixture was concentrated in vacuo and the residue was distilled under reduced pressure to give crude 7c contaminated with diisopropyl(dimethylsilyl)amine. Purification of the crude 7c by distillation $\left(72{ }^{\circ} \mathrm{C} / 24 \mathrm{mmHg}\right)$ gave $6 \mathrm{c}(9.96 \mathrm{~g}, 53 \%$ yield) which contains $10 \%$ of the $\alpha$-dimethylsilyl ester. $\quad$ 6c: ${ }^{1} \mathrm{H}$ NMR $\left(500 \mathrm{MHz}, \mathrm{C}_{6} \mathrm{D}_{6}\right) \delta 0.15(9 \mathrm{H}, \mathrm{d}, J=2.9 \mathrm{~Hz}), 1.35(3 \mathrm{H}, \mathrm{s})$, $4.69(1 \mathrm{H}, \mathrm{s}), 4.81(1 \mathrm{H}, \mathrm{s}), 4.92(1 \mathrm{H}$, sept, $J=2.8 \mathrm{~Hz}) ;{ }^{13} \mathrm{C}$ NMR $\left(125.8 \mathrm{MHz}, \mathrm{C}_{6} \mathrm{D}_{6}\right) \delta-2,31.3$, 45.3, 102.9, 153.7.

S-tert-Butyl (S)-5-Oxo-3-phenylhexanethioate (7a) (Typical Procedure for Asymmetric Michael Reaction, Table 1, entry 7). To a solution of $O$-(4-biphenoyl)- $N$-tosyl(L)-allo-threonine ${ }^{1}(45.4 \mathrm{mg}, 0.10 \mathrm{mmol})$ in $\mathrm{CH}_{2} \mathrm{Cl}_{2}(1 \mathrm{~mL})$ under argon atmosphere at room temperature was added dichlorophenylborane $(13 \mu \mathrm{L}, 0.10 \mathrm{mmol})$. After being stirred for 30 min, the mixture was concentrated in vacuo. To a solution of the resulting OXB 1a, silyl ketene acetal $\mathbf{6 b}$ (306 mg, $1.5 \mathrm{mmol})$, tert-butyl methyl ether $(88.2 \mathrm{mg}, 1.0 \mathrm{mmol})$ in $\mathrm{CH}_{2} \mathrm{Cl}_{2}(1 \mathrm{~mL})$ at 
$-78{ }^{\circ} \mathrm{C}$ were added a $\mathrm{CH}_{2} \mathrm{Cl}_{2}(1 \mathrm{~mL})$ solution of benzalacetone (5a) $(146 \mathrm{mg}, 1.0 \mathrm{mmol})$ and 2,6-diisopropylphenol (178 $\mathrm{mg}, 1.0 \mathrm{mmol}$ ) during $6 \mathrm{~h}$ by using a syringe pump. After the completion of the addition, the reaction mixture was stirred for $12 \mathrm{~h}$ at $-78^{\circ} \mathrm{C}$. The mixture was quenched by the addition of saturated aqueous $\mathrm{NaHCO}_{3}$ and filtered. The filtrate was extracted three times with hexane, dried $\left(\mathrm{Na}_{2} \mathrm{SO}_{4}\right)$, and concentrated in vacuo. The residue was dissolved in $1 \mathrm{~N} \mathrm{HCl}(2 \mathrm{~mL})-\mathrm{THF}(10 \mathrm{~mL})$ and the resulting solution was stirred at room temperature for 30 min. The mixture was poured into aqueous $\mathrm{NaHCO}_{3}$ and extracted three times with ether. The organic layers were dried $\left(\mathrm{Na}_{2} \mathrm{SO}_{4}\right)$ and concentrated in vacuo. Purification of the residue by flash chromatography $\left(\mathrm{SiO}_{2}, 5 \%\right.$ ethyl acetate in hexane) gave $217 \mathrm{mg}$ (78\%) of adduct $(S)-7 \mathbf{a}^{1}(94 \%$ ee).

Spectral data of new compounds in Table 2 are as follows.

S-tert-Butyl (S)-3-Benzyloxymethyl-5-Oxohexanethioate (7i): ${ }^{1} \mathrm{H}$ NMR (500 $\mathrm{MHz}, \mathrm{CDCl}_{3}$ ) $\delta 1.44(9 \mathrm{H}, \mathrm{s}), 2.11(3 \mathrm{H}, \mathrm{s}), 2.45-2.51(2 \mathrm{H}, \mathrm{m}), 2.55-2.62(2 \mathrm{H}, \mathrm{m}), 2.72(1 \mathrm{H}$, sept, $J=6.4 \mathrm{~Hz})$, $3.38(1 \mathrm{H}, \mathrm{dd}, J=5.7$ and $9.4 \mathrm{~Hz}), 3.48(1 \mathrm{H}, \mathrm{dd}, J=5.3$ and $9.4 \mathrm{~Hz}), 4.46(3 \mathrm{H}, \mathrm{s}), 7.25-7.35(5 \mathrm{H}$, $\mathrm{m}) ;{ }^{13} \mathrm{C}$ NMR $\left(125.8 \mathrm{MHz}, \mathrm{CDCl}_{3}\right) \delta 29.7,30.3,32.2,44.7,45.7,48.0,71.9,73.0,127.5,127.6$, 128.3, 138.2, 199.2, 207.4. Anal. Calcd for $\mathrm{C}_{18} \mathrm{H}_{26} \mathrm{O}_{3} \mathrm{~S}$ : C, 67.04; H, 8.13. Found: C, 66.70; H, 8.10. Ee determination (HPLC, Daicel Chiralpak AD-H, $1.0 \mathrm{~mL} / \mathrm{min}, 1 \%$ 2-PrOH in hexane) $(S) t_{1}=20.6 \mathrm{~min},(R) t_{2}=29.2 \mathrm{~min}$. The absolute stereochemistry was assumed by analogy.

S-tert-Butyl (S)-3-Methyl-5-oxoheptanethioate (7k): ${ }^{1} \mathrm{H}$ NMR $\left(500 \mathrm{MHz}, \mathrm{CDCl}_{3}\right) \delta 0.93(3 \mathrm{H}$, $\mathrm{d}, J=6.5 \mathrm{~Hz}), 1.00(3 \mathrm{H}, \mathrm{t}, J=7.3 \mathrm{~Hz}), 1.41(9 \mathrm{H}, \mathrm{s}), 2.23-2.55(7 \mathrm{H}, \mathrm{m}) ;{ }^{13} \mathrm{C}$ NMR $(125.8 \mathrm{MHz}$, $\left.\mathrm{CDCl}_{3}\right) \delta 7.7,19.7,27.2,29.7,36.3,47.9,48.4,50.7,199.3,210.3$. Ee determination (HPLC, Daicel Chiralpak AD-H, $0.5 \mathrm{~mL} / \mathrm{min}, 1 \% 2-\mathrm{PrOH}$ in hexane) $(S) t_{1}=23.7 \mathrm{~min},(R) t_{2}=24.6 \mathrm{~min}$. The absolute stereochemistry was assumed by analogy.

S-tert-Butyl (S)-5-Oxo-3-phenylnonanethioate (7l): ${ }^{1} \mathrm{H}$ NMR $\left(500 \mathrm{MHz}, \mathrm{CDCl}_{3}\right) \delta 0.83(3 \mathrm{H}$, t, $J=7.4 \mathrm{~Hz}), 1.16-1.24(2 \mathrm{H}, \mathrm{m}), 1.38(9 \mathrm{H}, \mathrm{s}), 1.42-1.48(2 \mathrm{H}, \mathrm{m}), 2.21-2.34(2 \mathrm{H}, \mathrm{m}), 2.71(1 \mathrm{H}$, dd, $J=7.3$ and $14.7 \mathrm{~Hz}), 2.75-2.86(3 \mathrm{H}, \mathrm{m}), 3.71(1 \mathrm{H}$, quintet, $J=7.2 \mathrm{~Hz}), 7.17-7.20(3 \mathrm{H}, \mathrm{m})$, 7.25-7.29 (2H, m); $\left.{ }^{13} \mathrm{C} \mathrm{NMR} \mathrm{(125.8} \mathrm{MHz,} \mathrm{CDCl}_{3}\right) \delta$ 13.7, 22.1, 25.6, 29.6, 38.0, 42.9, 48.0, 48.1, 50.3, 126.7, 127.4, 128.5, 142.7, 198.5, 209.0. Anal. Calcd for $\mathrm{C}_{19} \mathrm{H}_{28} \mathrm{O}_{2} \mathrm{~S}$ : C, 71.20; $\mathrm{H}, 8.81$. Found: C, 71.13; H, 8.86. Ee determination (HPLC, Daicel Chiralpak AD, $1.0 \mathrm{~mL} / \mathrm{min}, 1 \%$ 2-PrOH in hexane) $(S) t_{1}=11.7 \mathrm{~min},(R) t_{2}=17.4 \mathrm{~min}$. The absolute stereochemistry was assumed by analogy. 


\section{Reference}

(1) Wang, X.; Adachi, S.; Iwai, H.; Takatsuki, K.; Fujita, M.; Kubo, M.; Oku, A.; Harada, T. J. Org. Chem. 2003, 68, 10046. 

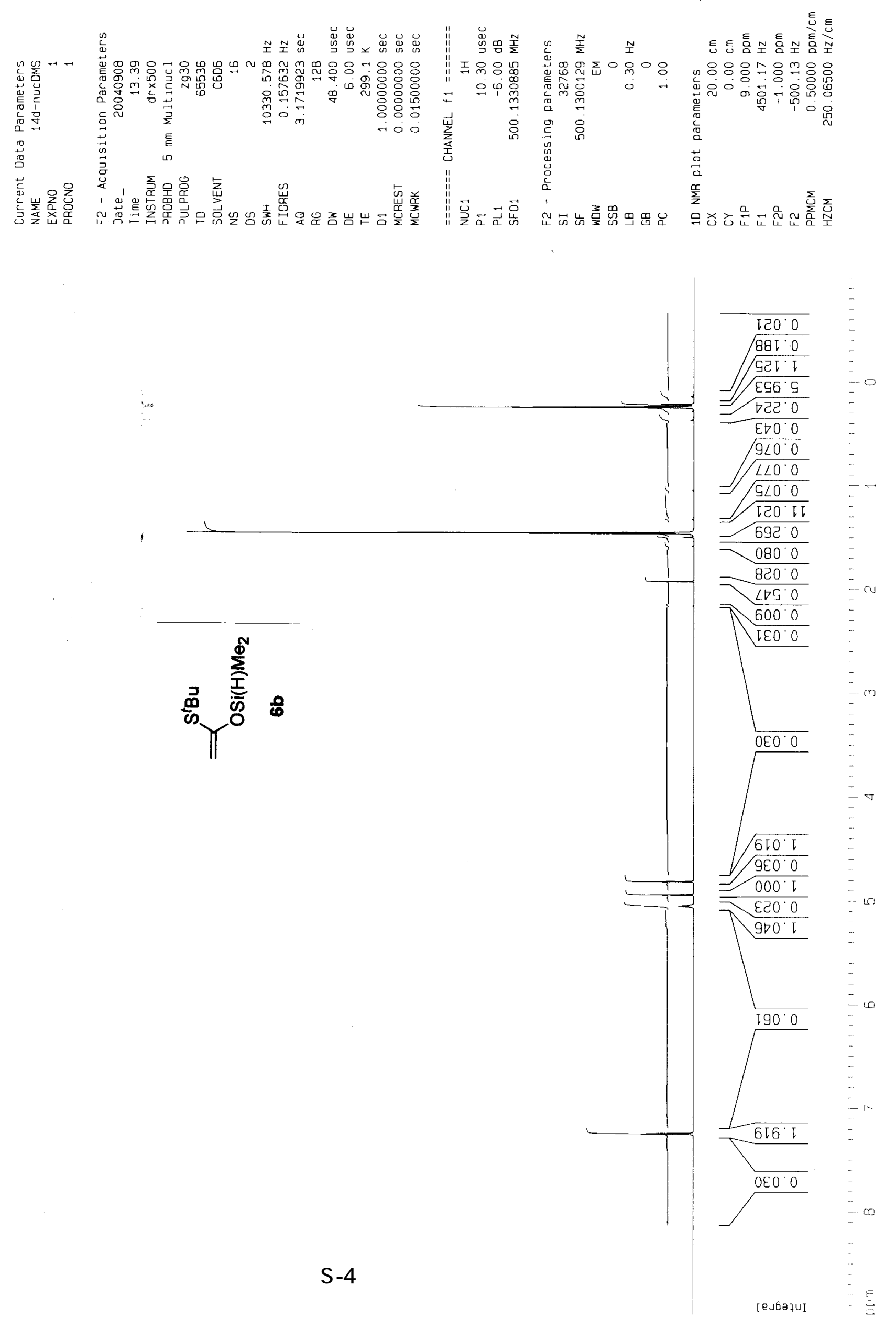

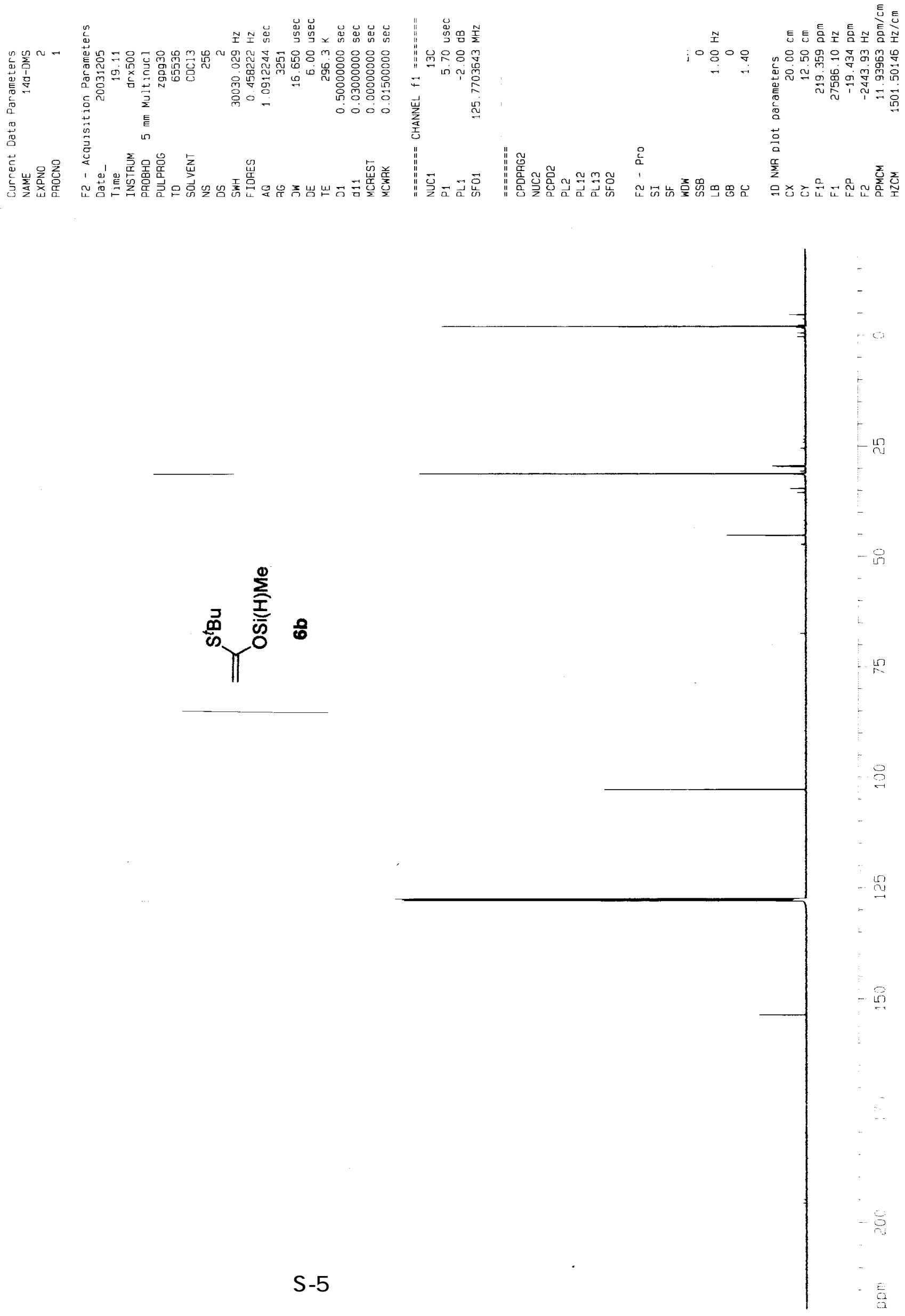

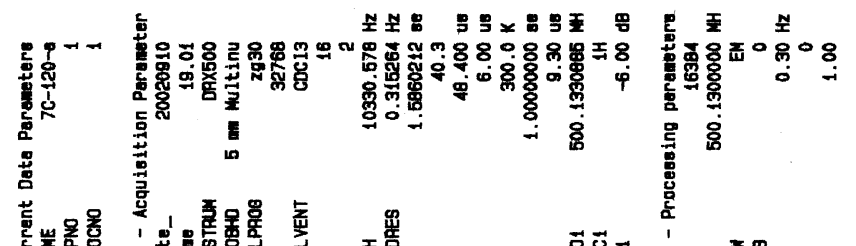

른 믐소 몸소 뭄소

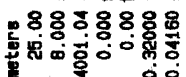

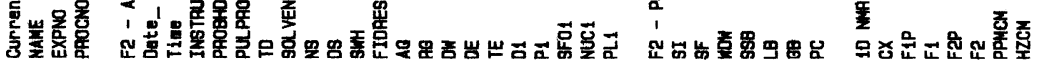
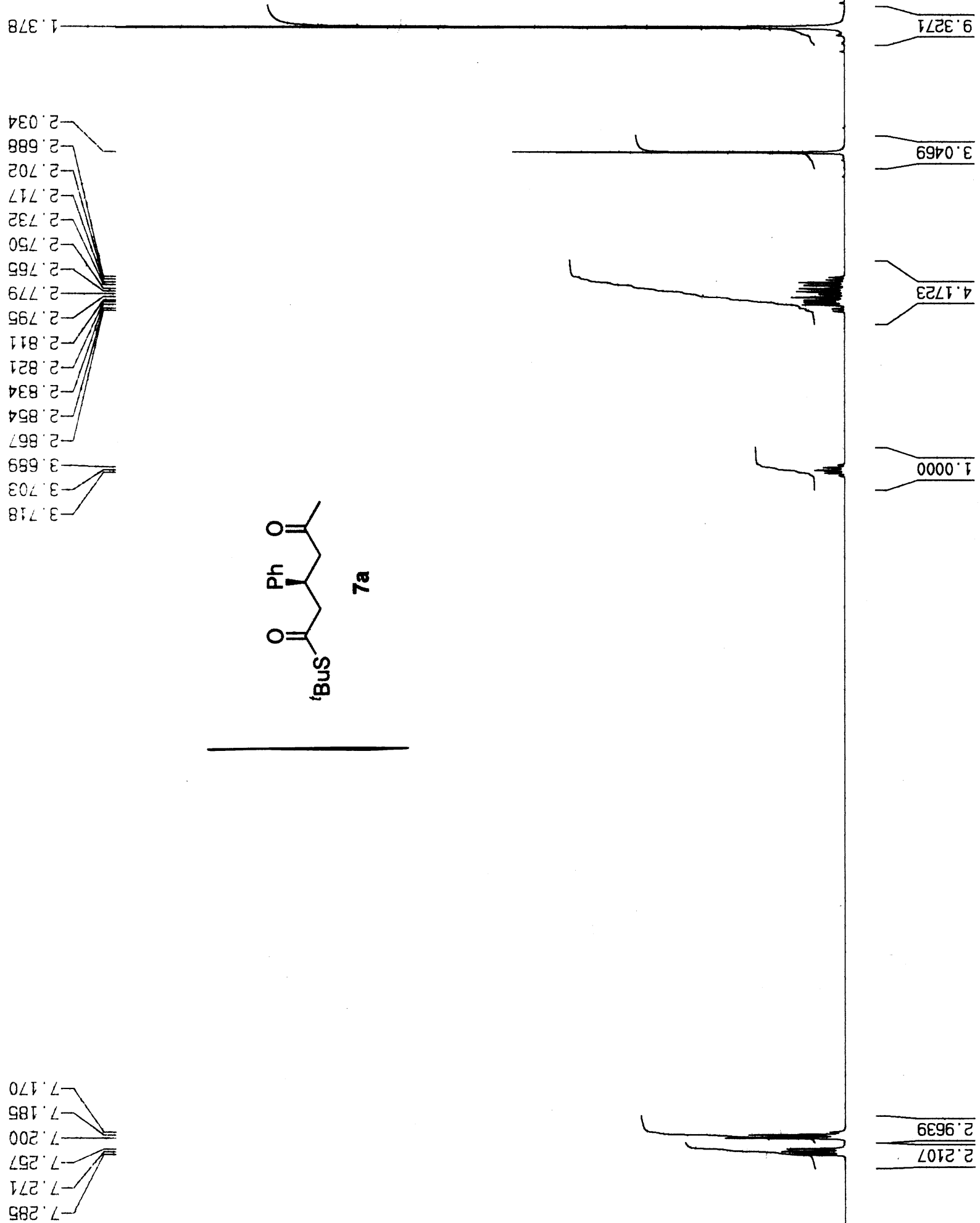

$0000^{\circ}$

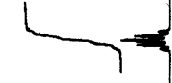




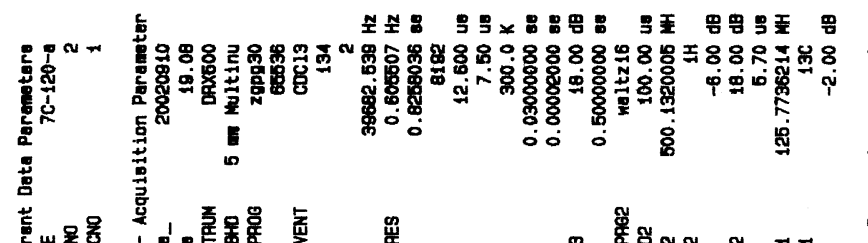

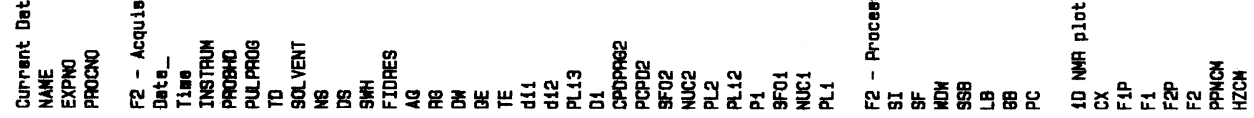

89.62

$\nabla E^{\circ} O E$

$\nabla 6^{\circ} \angle E$

70.87

$\angle 6{ }^{\circ} 8 \nabla$

or $6 \nabla$

$9 E^{\prime}$ OS

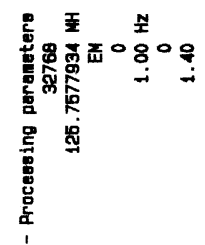

늠소늠소늠소

年8

त

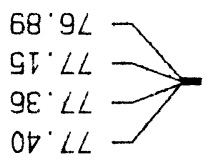

8L'9टโ-

96.9टT-

II'LCT

$9 E^{\circ} \angle 2 I$

$E G^{\circ} \angle 2 I$

टE'8टा-

$\varepsilon G^{\circ} 8 \mathrm{I} \downarrow$

ट9'ट॰

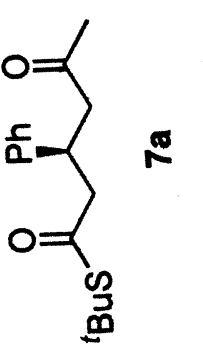



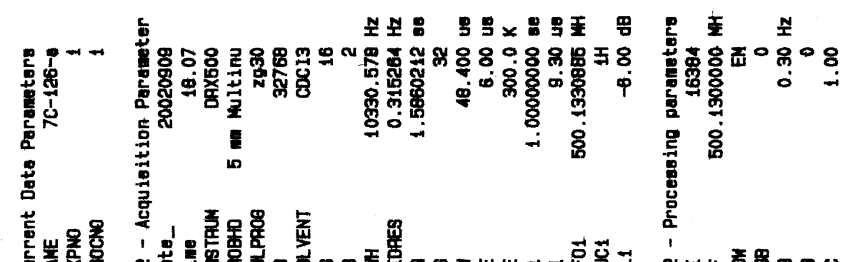

통음소음소음소

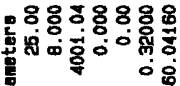

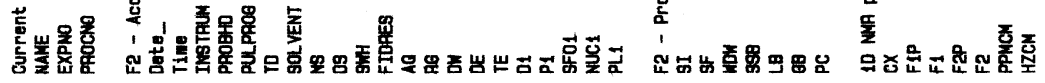

EOt

$900^{\circ} \mathrm{C}$

GOE' $\mathrm{C}$

$289^{\circ} \mathrm{C}$

$969^{\circ} \mathrm{C}$

IL $\angle$ '己]

$G \mathcal{G} L 2]$

Ob $L \cdot c 7$

$0 G L ' Z-$

GGL'己

$99 L ' \mathrm{C}$

$0 L L \cdot 2$

$E 8 L \cdot \mathrm{C}-$

66L ट⿱㇒冋

218.5-

828 ' ट-

$678^{\circ} \mathrm{C}-$

298 ' $\mathrm{C}-$

$\nabla 99^{\circ} \mathrm{E}$

$8 \angle 9^{\circ} \varepsilon$

$\varepsilon 69^{\circ} \varepsilon-$

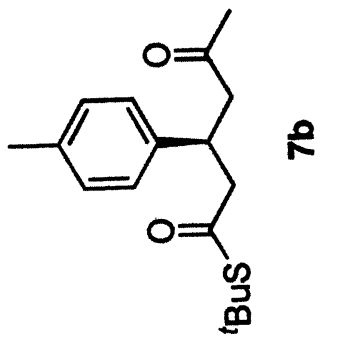

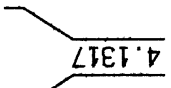
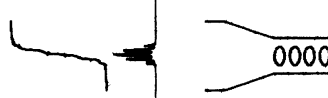

$\varepsilon 60^{\circ}\llcorner$ 


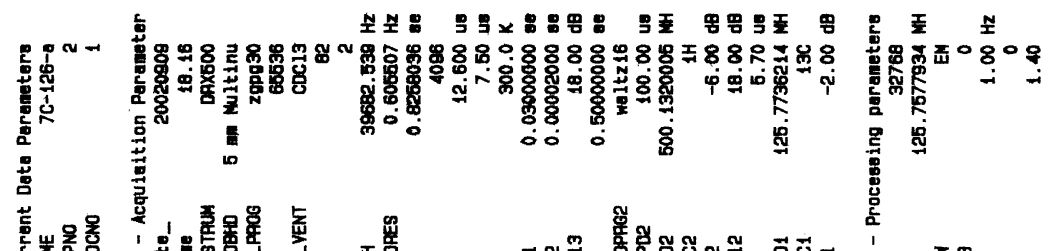

튼음소믐노믐소

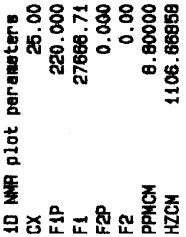

10.12

$-$

$99^{\circ} 62$

$\nabla \varepsilon^{\circ} O E$

$19 \cdot \angle E$

70.85

$80^{\circ} 67$

$\nabla 9^{\circ} \circ g_{-}$

$\angle 8^{\circ} 9 L$

$\varepsilon \tau ' L L$

$8 \varepsilon^{\prime} \angle L>$
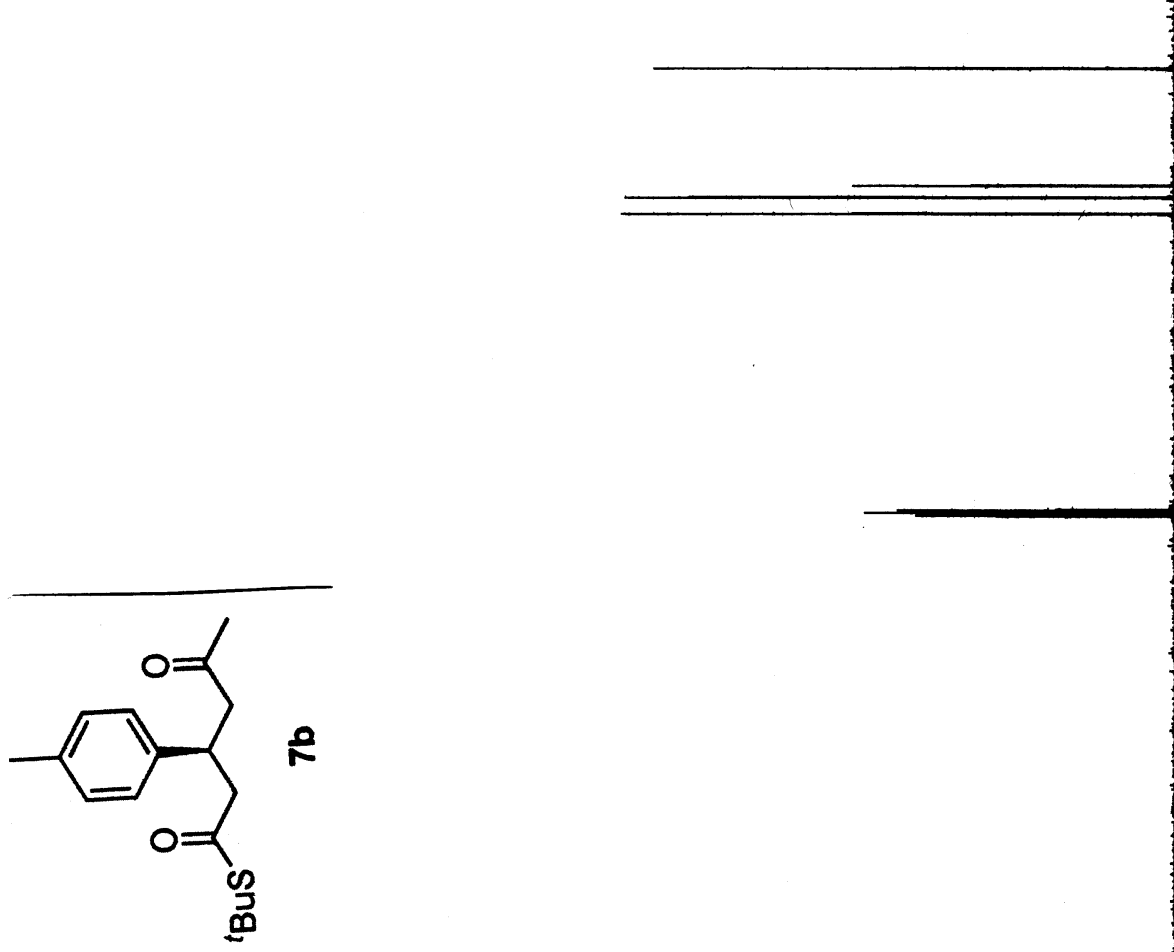

8I'LCID

टटं6己F-

Eट $9 E I-$

Ig $E E T$

R 

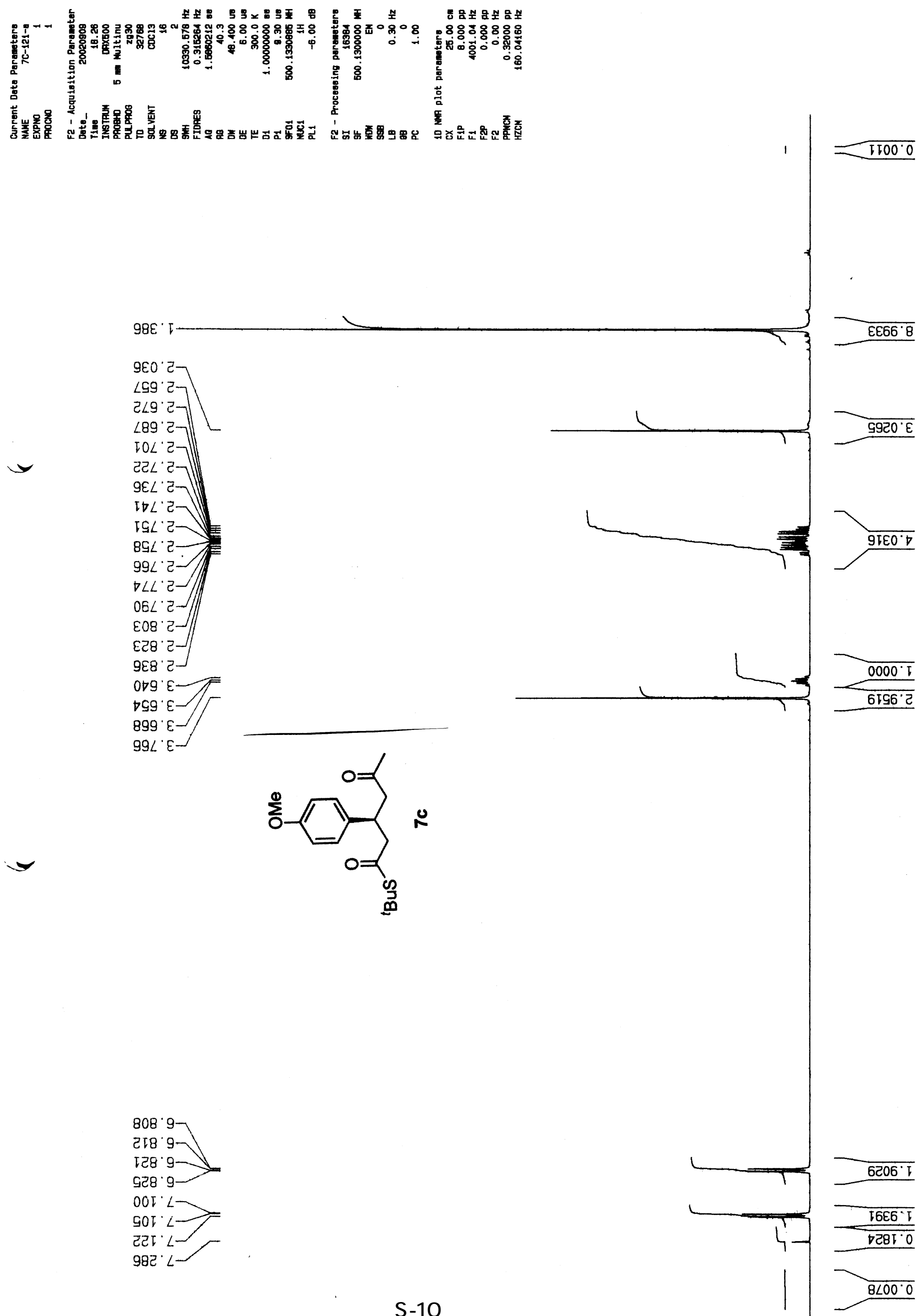

6म96 


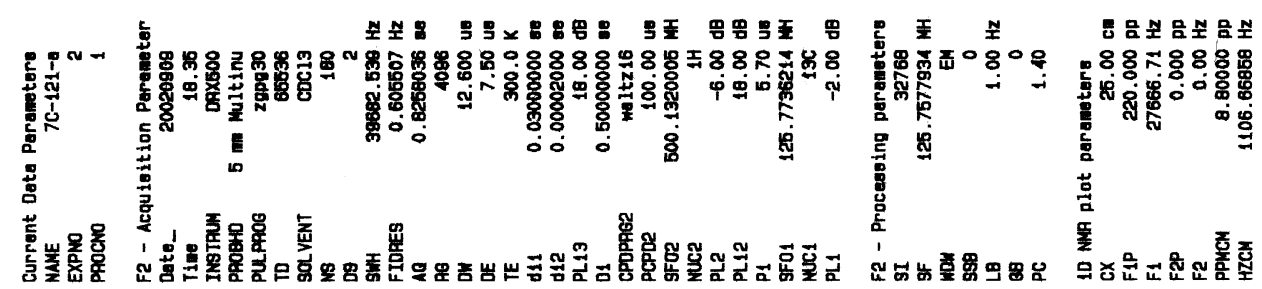

II $\nabla T$

$59 \cdot 52$

$E 9^{\circ} \theta \mathrm{C}$

$G \varepsilon^{\circ} \circ \varepsilon$

Gg. $T E$

$8 C^{\prime} \cdot \angle E$

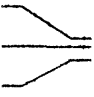

$\nabla 0.87$

Iट.67

29.09

SI. 99

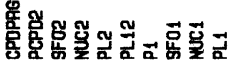

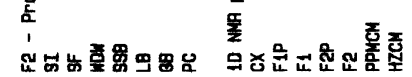

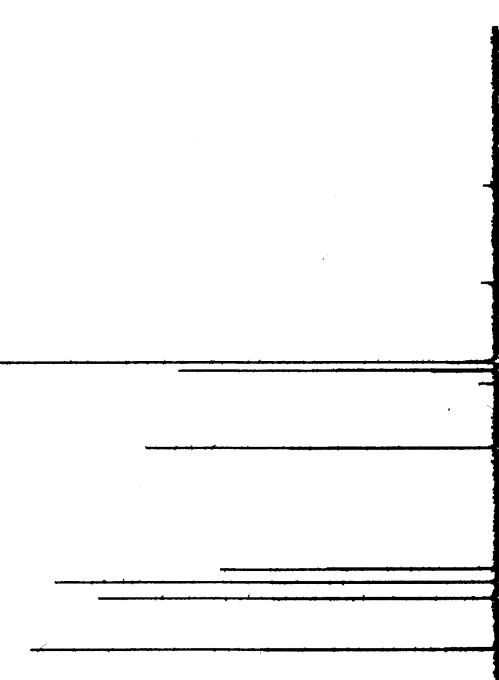

$\varepsilon 8^{\prime} 9 L$

$80^{\circ} \mathrm{LL}$

$\nabla E \angle L$

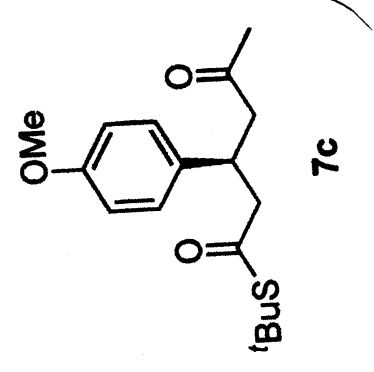

$\angle 8^{\prime} E I \tau$

IE' Bटโ

$\left\lceil 9^{\circ} \nabla \varepsilon\right.$

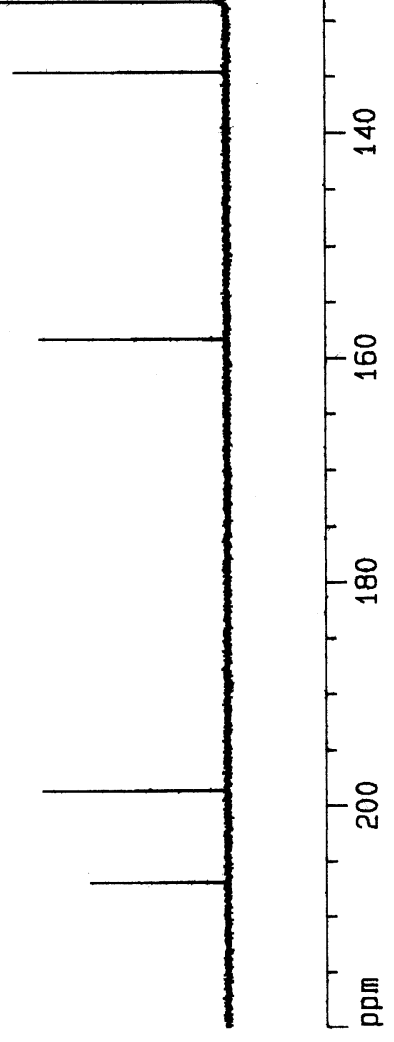

1 wdd 


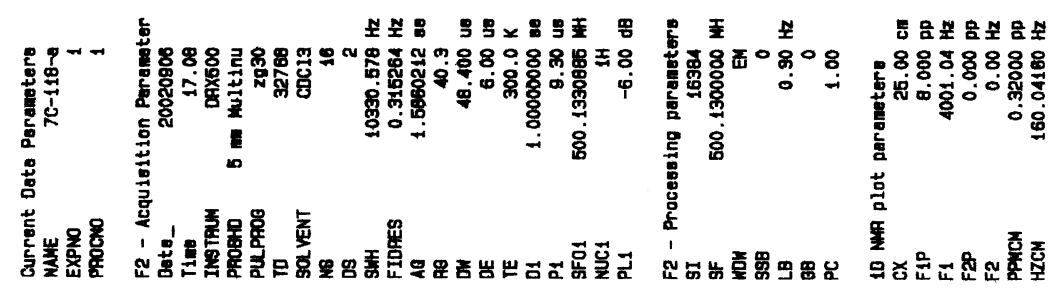

โ9E

$=000$.

$\nabla \varepsilon 0^{\circ} \mathrm{Z}$

$6 \varepsilon 9^{\circ} \vec{c}$

$\nabla 99^{\circ} \mathrm{C}$

699 ' 27

๑89. 2

$\varepsilon ट L ' \partial$

$\angle E$

टSL'

$\angle G L^{\prime} \mathrm{C}$

$\angle 9 L \cdot 2$

ELL' ट

96L' 2

808 . $2-$

0ह8.

टฤ8 ट-

$899^{\circ} \varepsilon$

ट $\angle 9^{\circ} \varepsilon-$

$\angle 89^{\circ} \varepsilon$
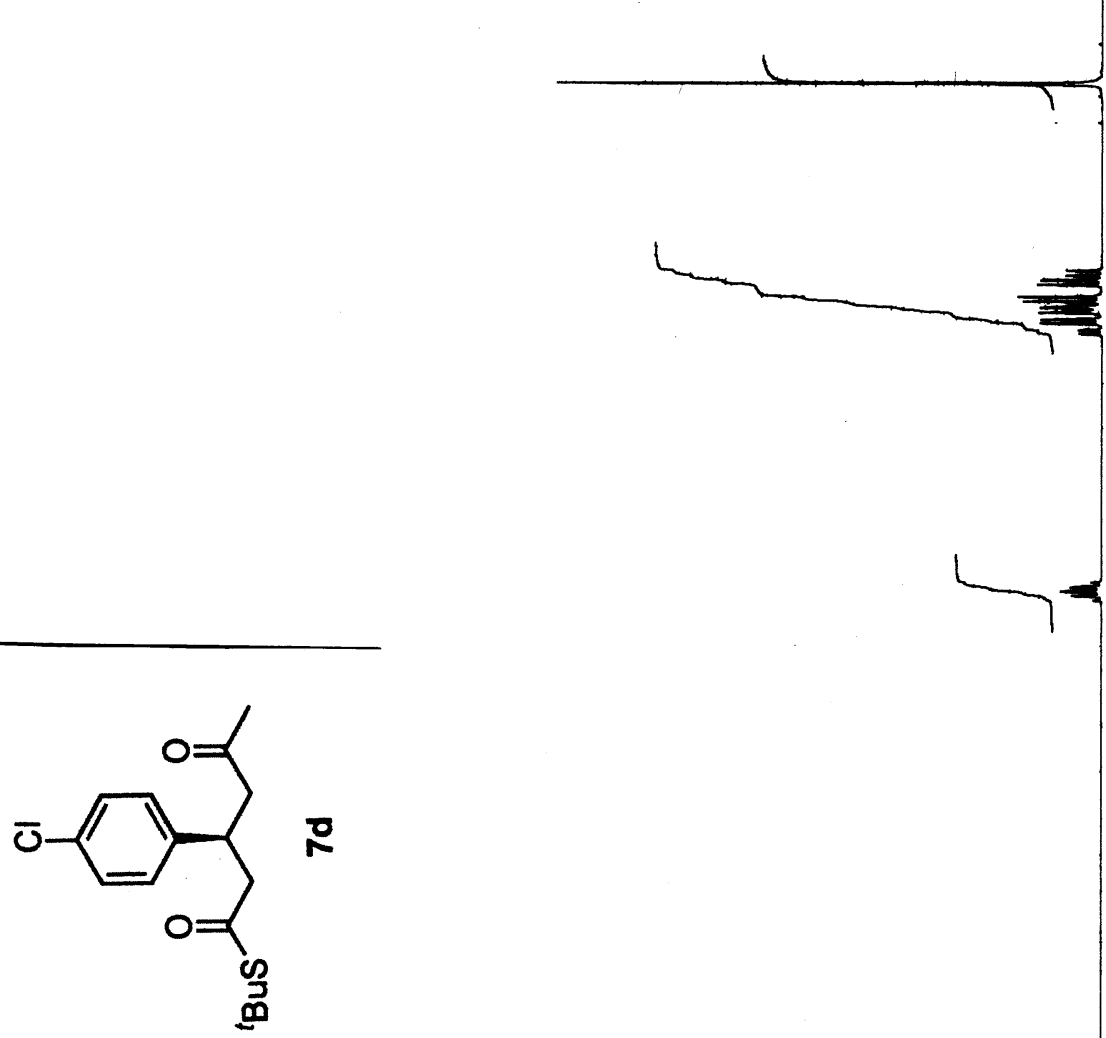

$9800^{\circ}$
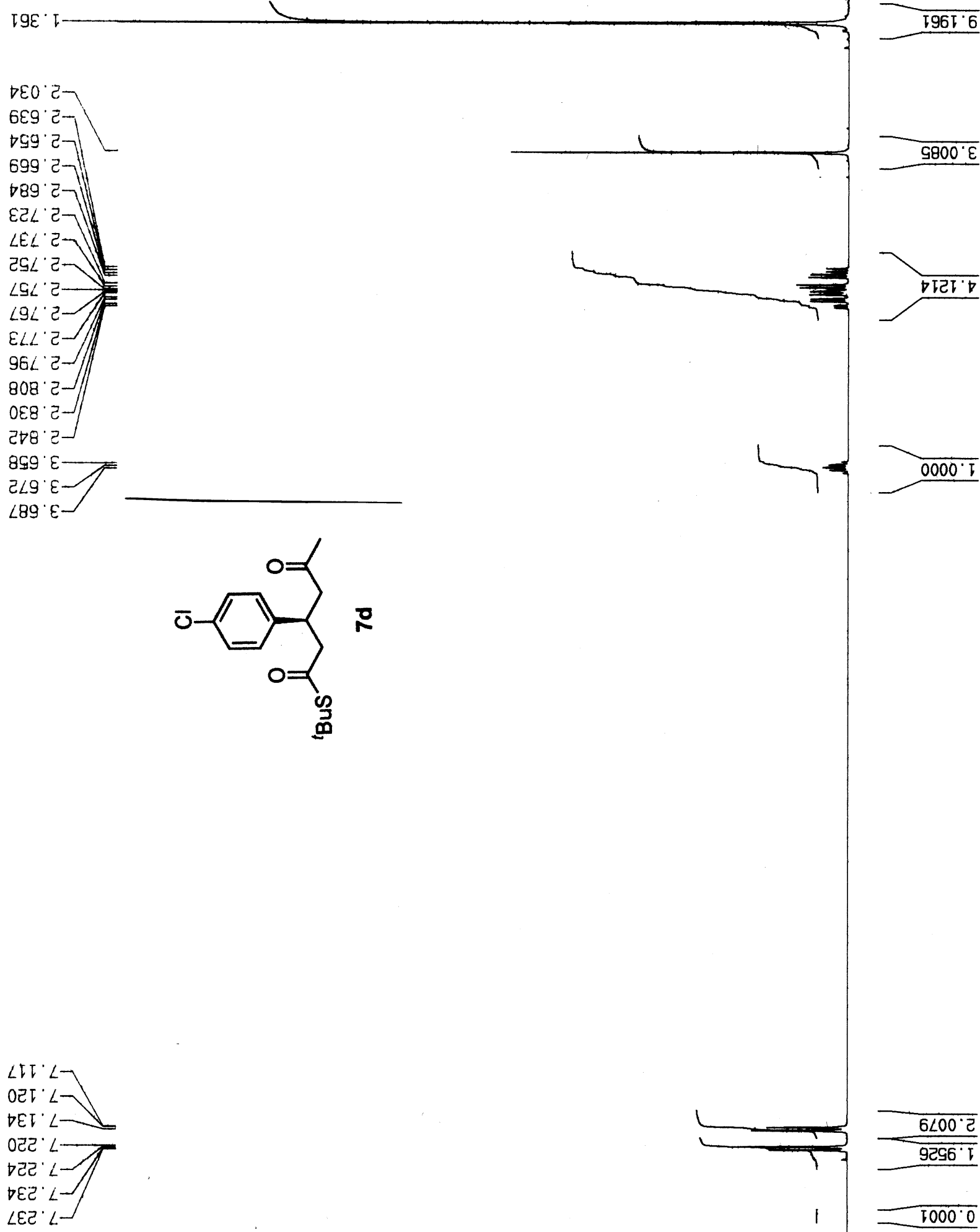

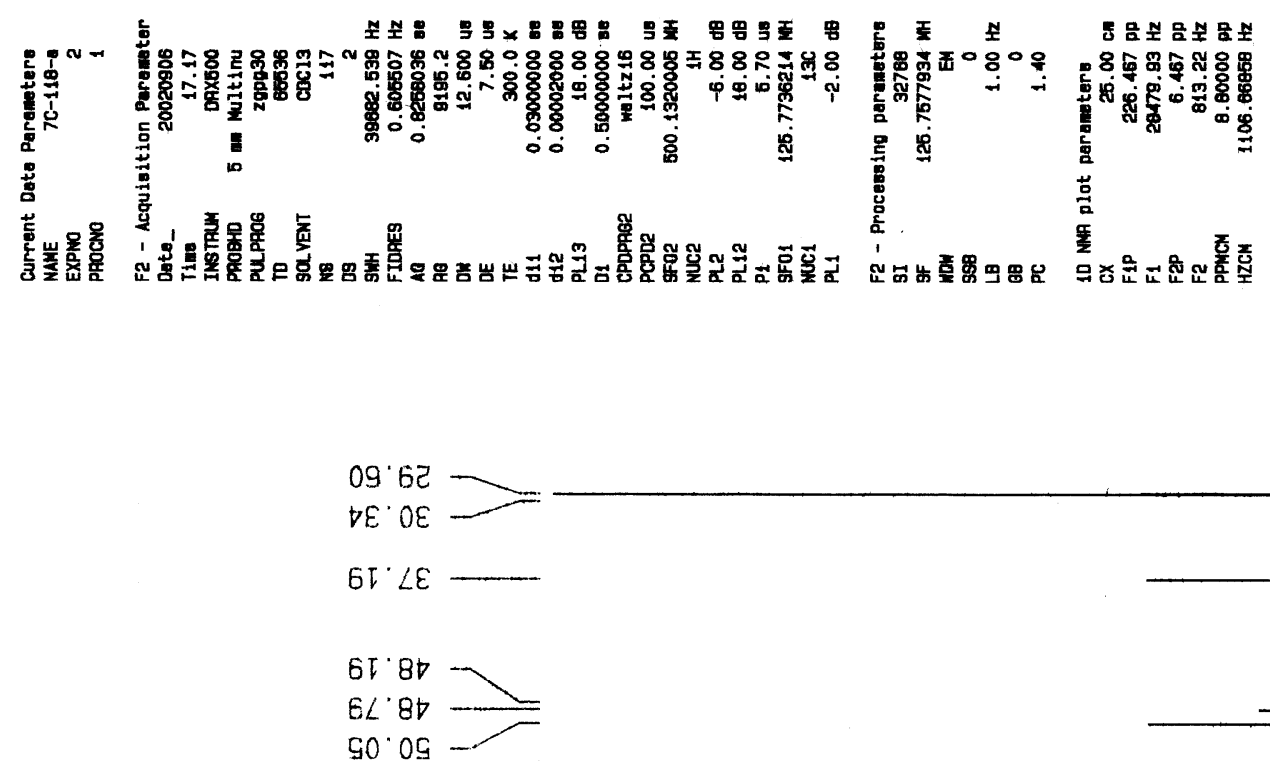

$98 \cdot 9 L$

II' $L L$

$\angle E^{\prime} L L$
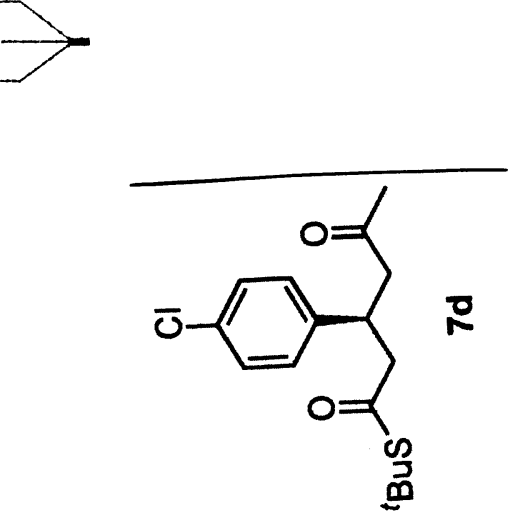

19.8टT

ट8. 8 ट

$\varepsilon 0$.

$\angle E \cdot ट E T-$

9「饦— 

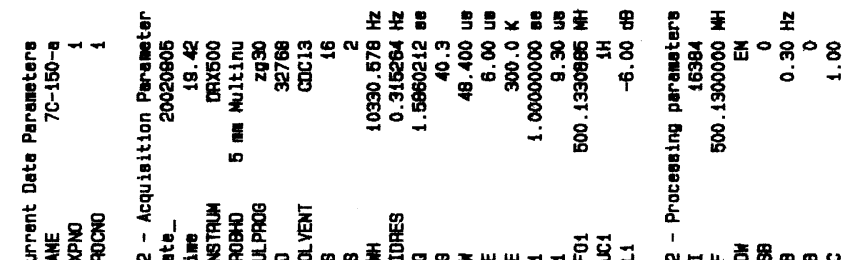

통믐소음소늠소

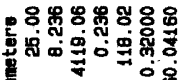

营

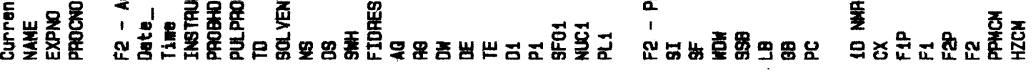
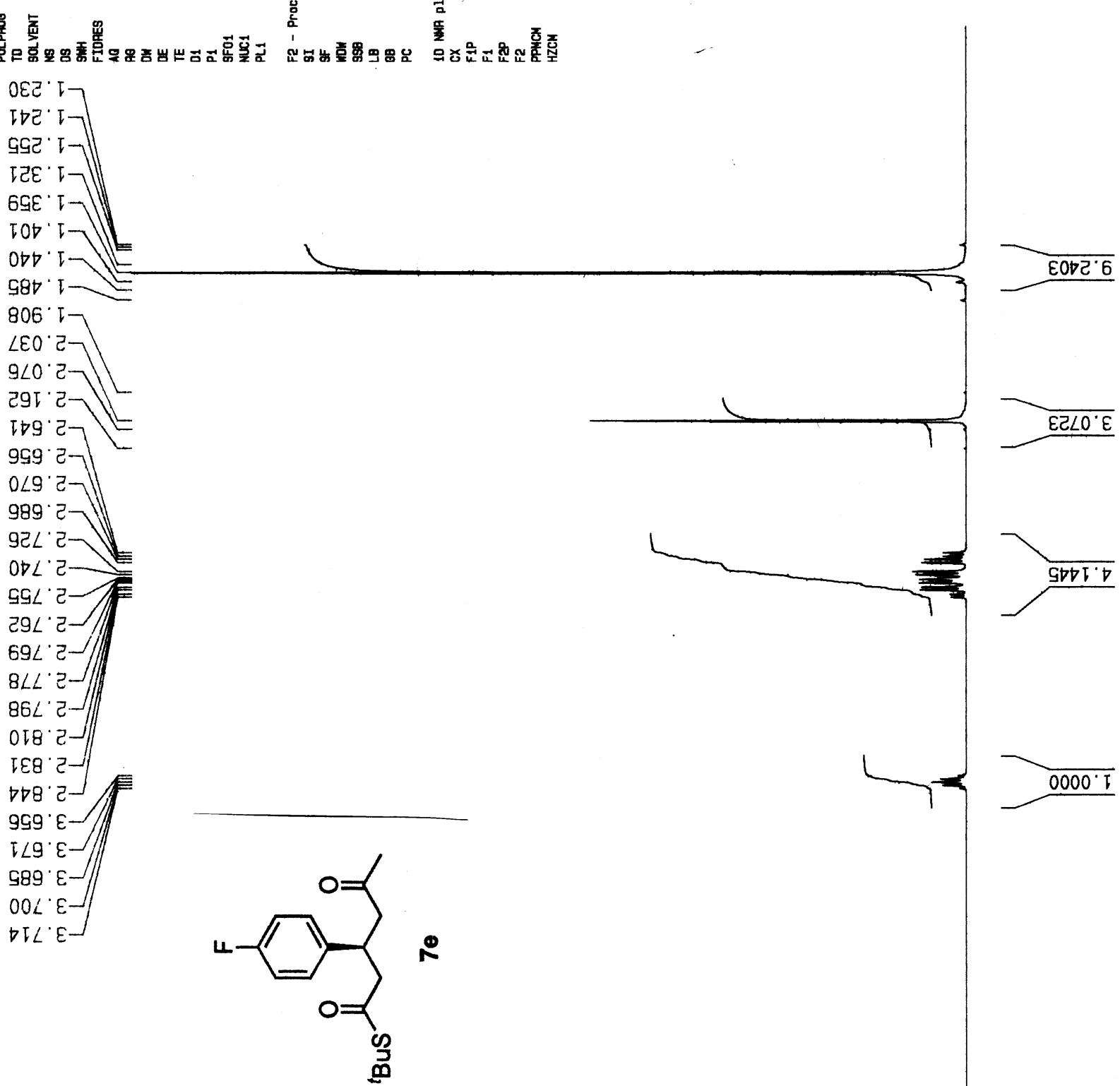

$$
989
$$

$92 L ' \bar{c}$

$O D L 2$

$291 \cdot 2$

$69 L^{\circ} \mathrm{C}-$

$8 L L \cdot 5-$

$\nabla \nabla \theta^{\circ} \mathrm{C}$

$\tau \angle 9^{\circ} \varepsilon$

$\varsigma 89^{\circ} \varepsilon-$

$00 \angle ' \varepsilon$

$\nabla I L \cdot E-$

品

$\angle B L ' 9$

IEE' 9

$8 \nabla 6.9$

996.9

टౌ厂' $L$

EGT' $\angle$

$69 T \cdot \angle-/ F$

$69 \mathrm{I}^{\circ} \mathrm{L}$

$58 \mathrm{LC} \cdot$

6EE $\angle-$
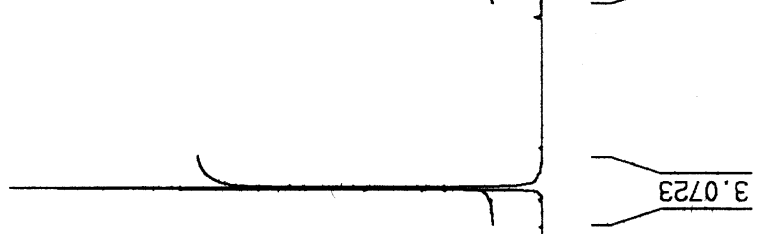

$-\infty$

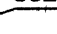



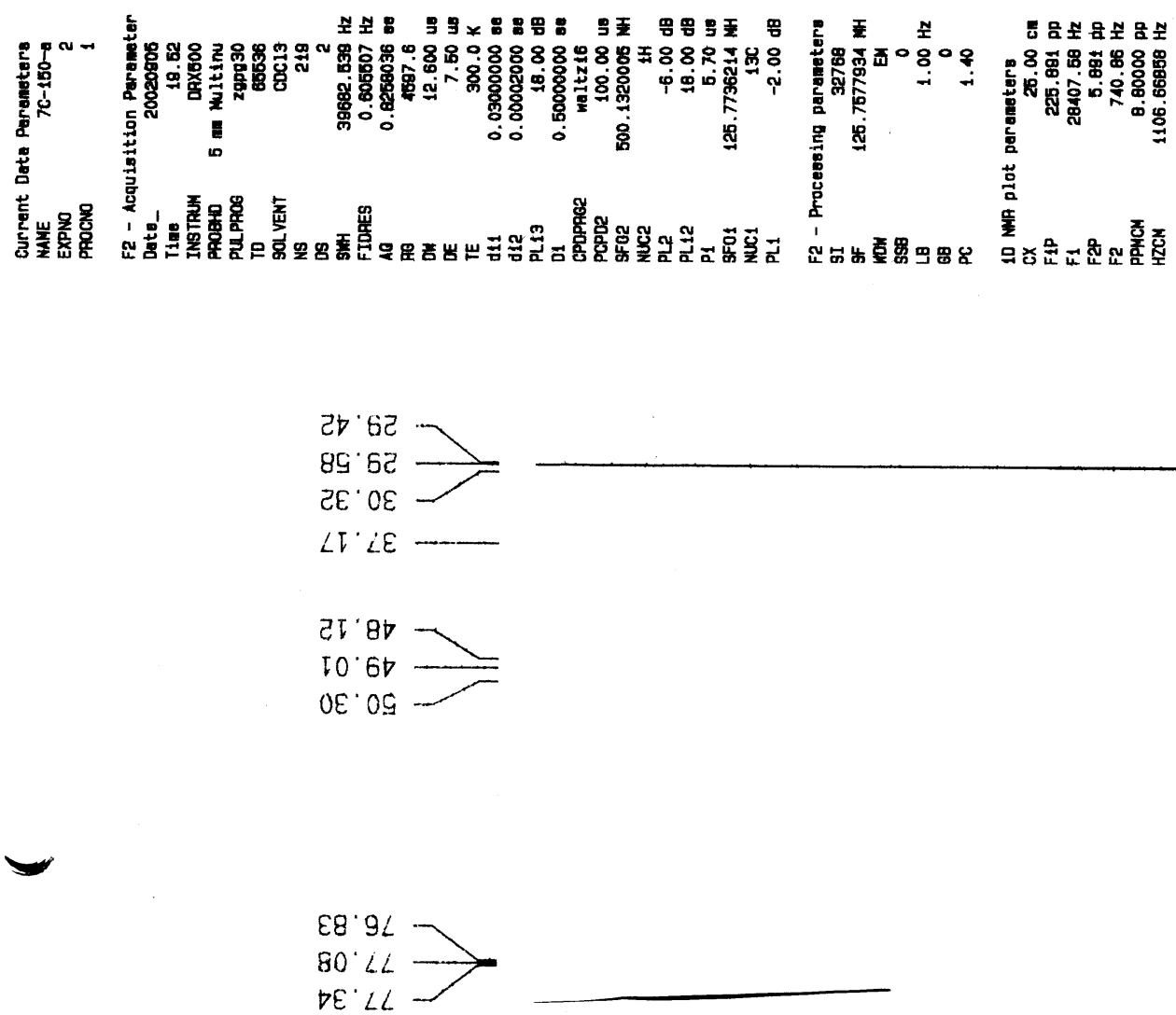

8I GIT-

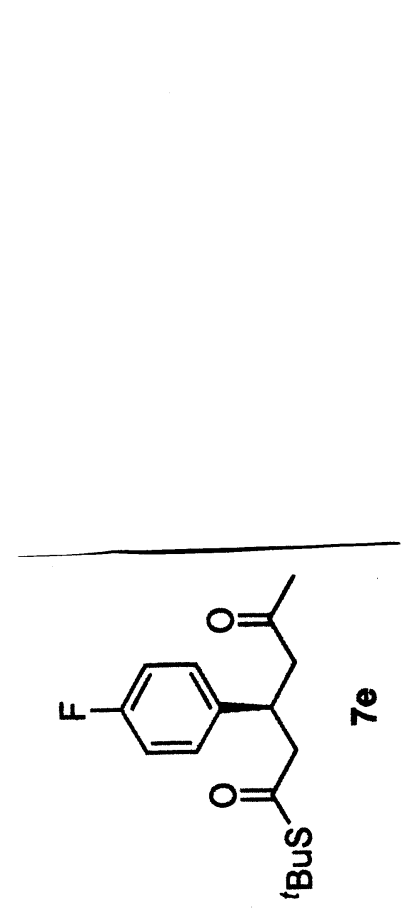

GE'GIT

$\angle 8 \cdot 82 \tau$

E6. $\mathrm{gCT}$

$9 e^{\prime} 8 \varepsilon T$

6己' $8 E I$

$\angle G^{\circ} 091$

I9.29:-

(n) 

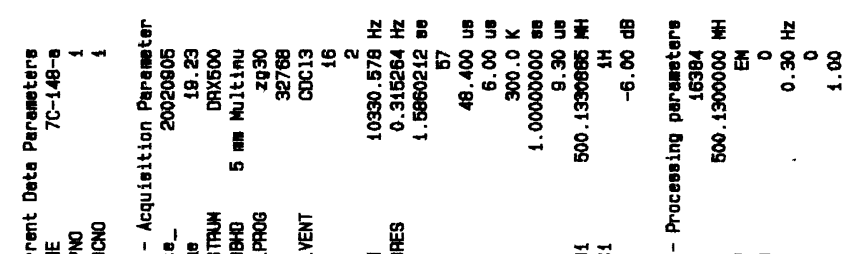

틍믈소음소 응소
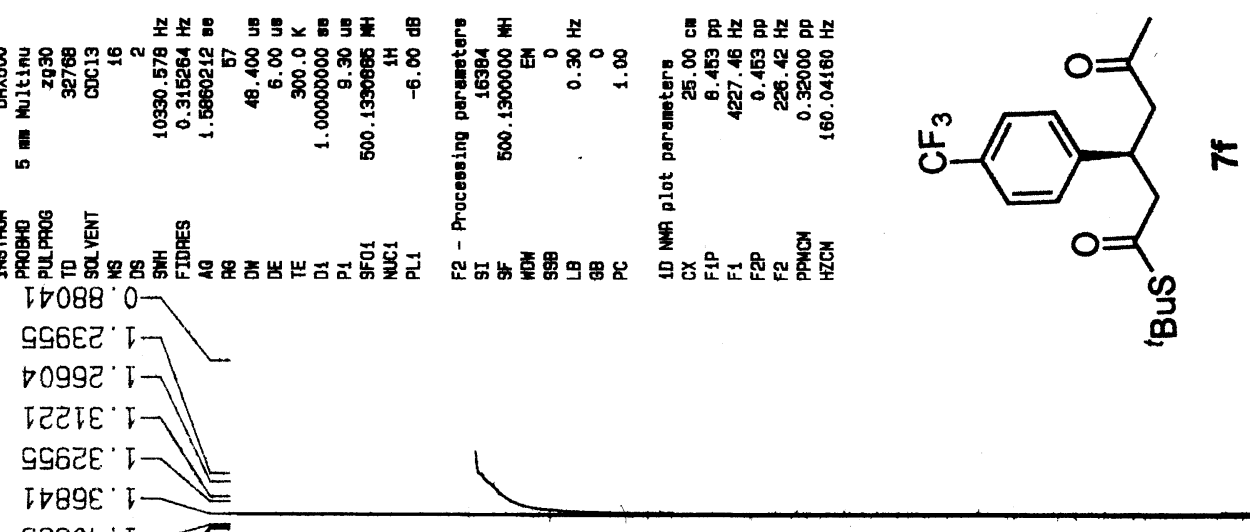

$$
\text { GGEOt }
$$

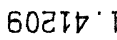

8I9t大

ट $\angle \nabla 60^{\circ} \mathrm{T}$

$01890^{\circ} \mathrm{C}$

$9 \angle \nabla 69^{\circ} \mathrm{C}-$

E660L'已-

$\nabla 8 \nabla ट L \cdot c-$

666EL' 2

$008 \angle L ' Z$

$\nabla \nabla 26 \angle ' 2$

$66 \angle 08^{\circ} \mathrm{C}$

ВEटटQ 'ट-

$\checkmark$ I9ट8'ट-

हटटऽg. ट-

90ट98. ट-

$99 \square \angle 8^{\circ} \mathrm{C}-$

$\nabla \nabla 968^{\circ} \mathrm{C}$

$\nabla 0606^{\circ} \mathrm{C}$

$\angle G D S \angle{ }^{\prime} \varepsilon$

$\varepsilon[69 L ' \varepsilon-$

ISE8L $\varepsilon-$

$O \angle L 6 L{ }^{\prime} \varepsilon-$

टटटा8' $\varepsilon$

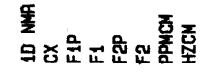

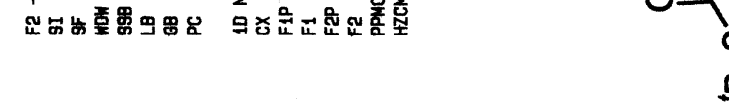

$k$

6ะ8६E' 9

GEOLE'9-

G $9 \angle 9 \cdot 9$

$9 \$ 80 L \cdot 9$

OESBट ' $L$

SODटE $\angle 7$

9LO॰ $\varepsilon^{\circ} \angle$

BES9E $\angle$

$\angle Z E G D^{\circ} \angle$

$\angle G 690^{\circ} \angle$

GटLGD' $\angle$

IEIEG : $\angle$

टG $\triangle \nabla G \cdot \angle$

$\nabla 2 \angle 89^{\circ} \angle$

$\forall \angle$ I69 $\angle$

ILLOL'L
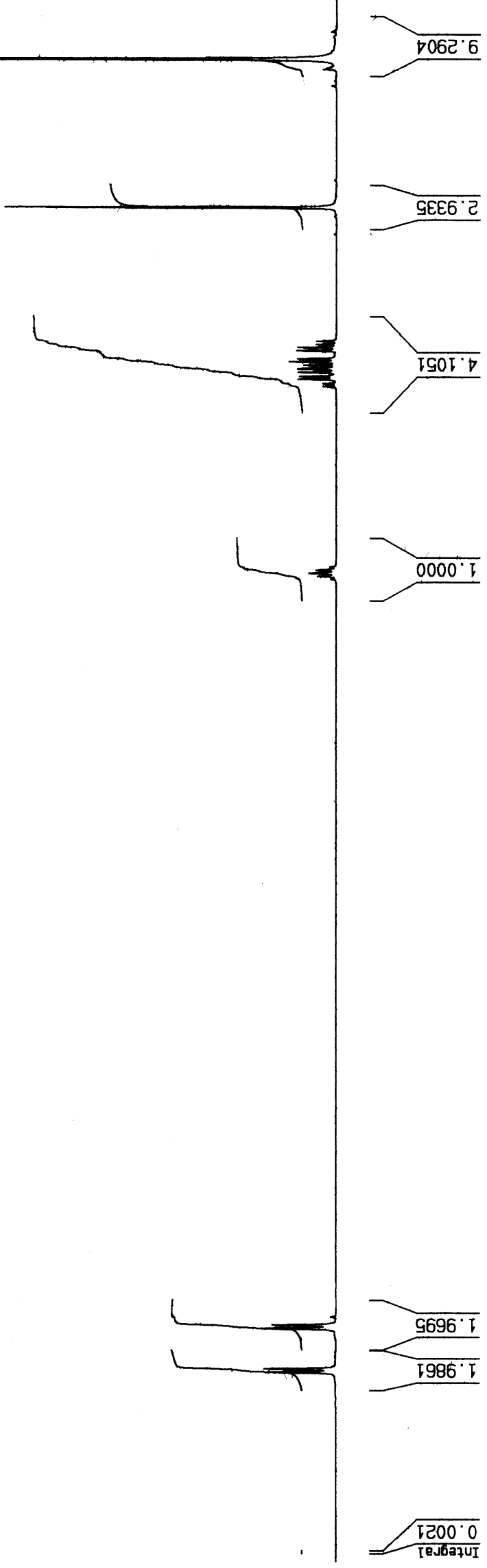

$9696^{\circ} \mathrm{T}$ 

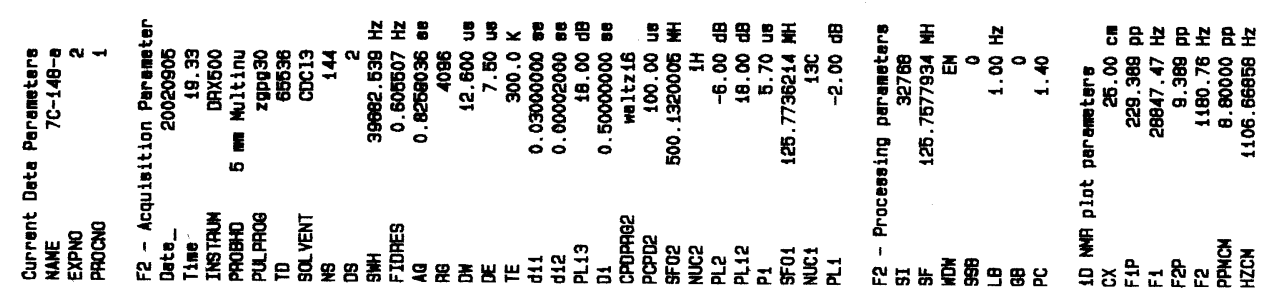

$87^{\prime} 62$

G9.6己

$\angle C^{\prime} O E$

$8 \nabla^{\circ} \angle \varepsilon$

$\angle 2 \cdot 8 D$

Gg. 87

$9 L^{\circ} 6 \nabla$

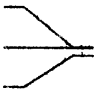

$-$

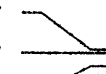

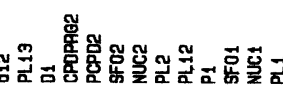

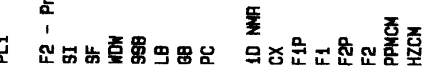
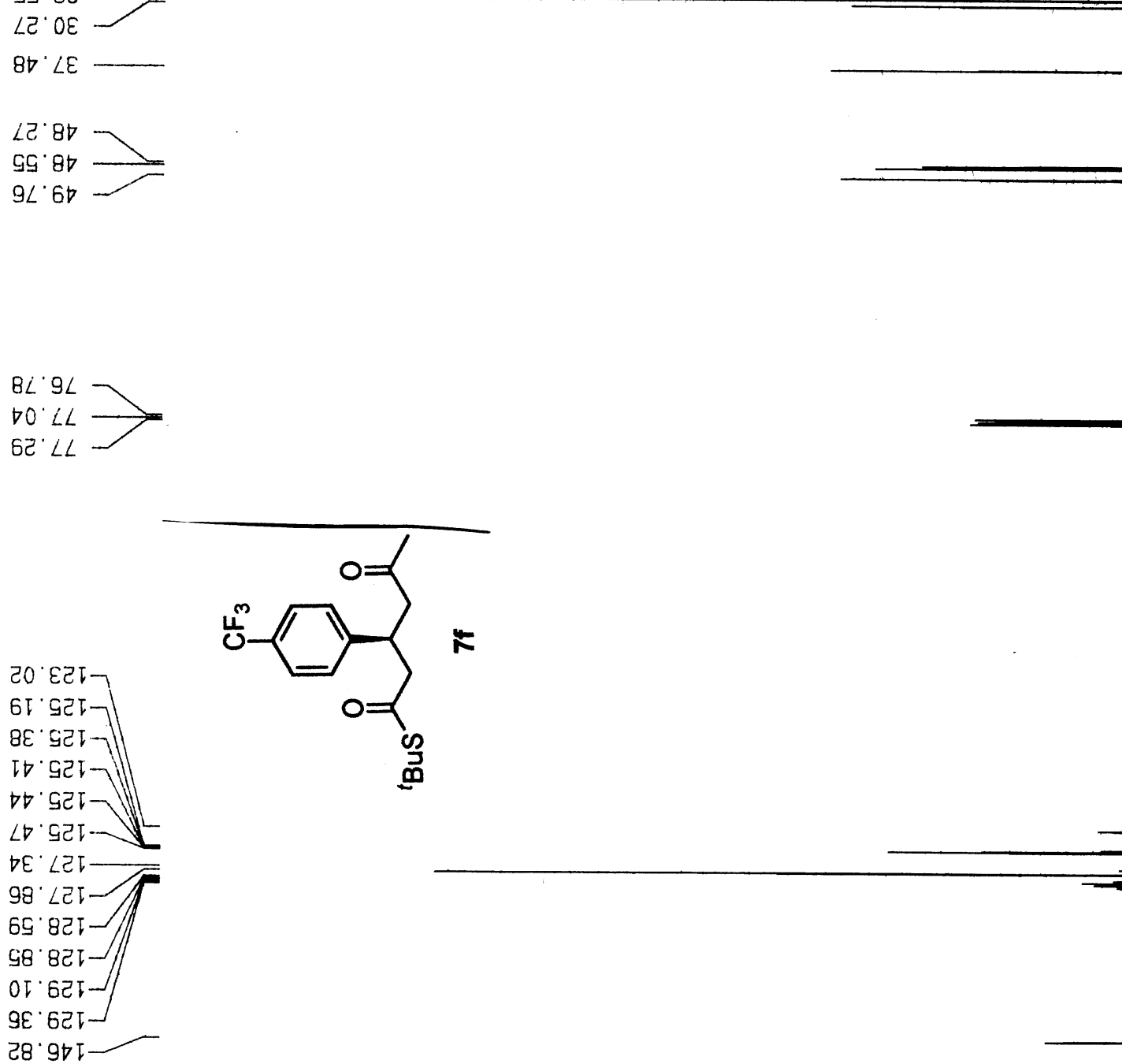

$\nabla 0.86 \tau$

$68 \cdot 902-$ 

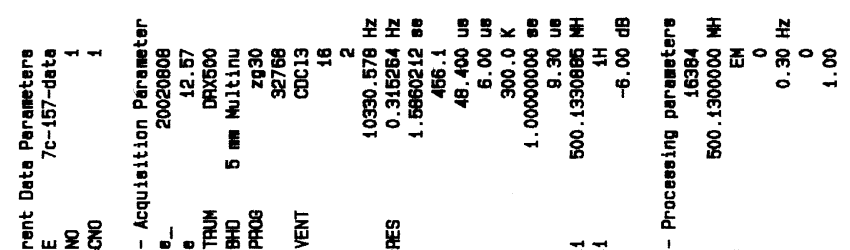

통음소은소믐소

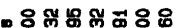

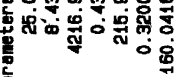

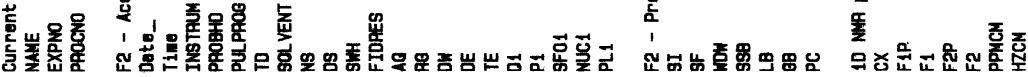

698

6GE' $[7]$

$\angle 8 E^{\prime} \cdot T$

$\angle 己 \nabla \cdot \neg$

$\varepsilon \nabla \nabla$

$96 b^{\circ} T \cdot$

EIG.T-

I09. 1

$6 E 0^{\circ} \mathrm{s}$

$90 T^{\prime} \mathrm{C}$

$\varepsilon \varepsilon \digamma$ '

$\angle O L \cdot 27 L$

टटटL'ट-

9EL'ट-

ISL'

$86 L^{\prime} \mathrm{C}>$

टा8'ट

$8 ट 8^{\circ} \mathrm{C}$

ट†8

$678^{\circ} 2$

$\nabla 98^{\circ} \mathrm{z}-$

$868^{\circ} \mathrm{C}-\mathrm{J}$

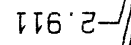

टह6. ट-

Sक6 2 -

$986.2-$

$98 \varepsilon^{\circ} \varepsilon-$

$G \angle L \cdot \varepsilon-$

$06 L^{\prime} \varepsilon-$

$\nabla 08^{\circ} \varepsilon-$

$818^{\circ} \varepsilon$

$\varepsilon \varepsilon 8^{\circ} \varepsilon-$

$989^{\circ} \circ-$

$\angle E 8^{\circ} \nabla$

s9t's-
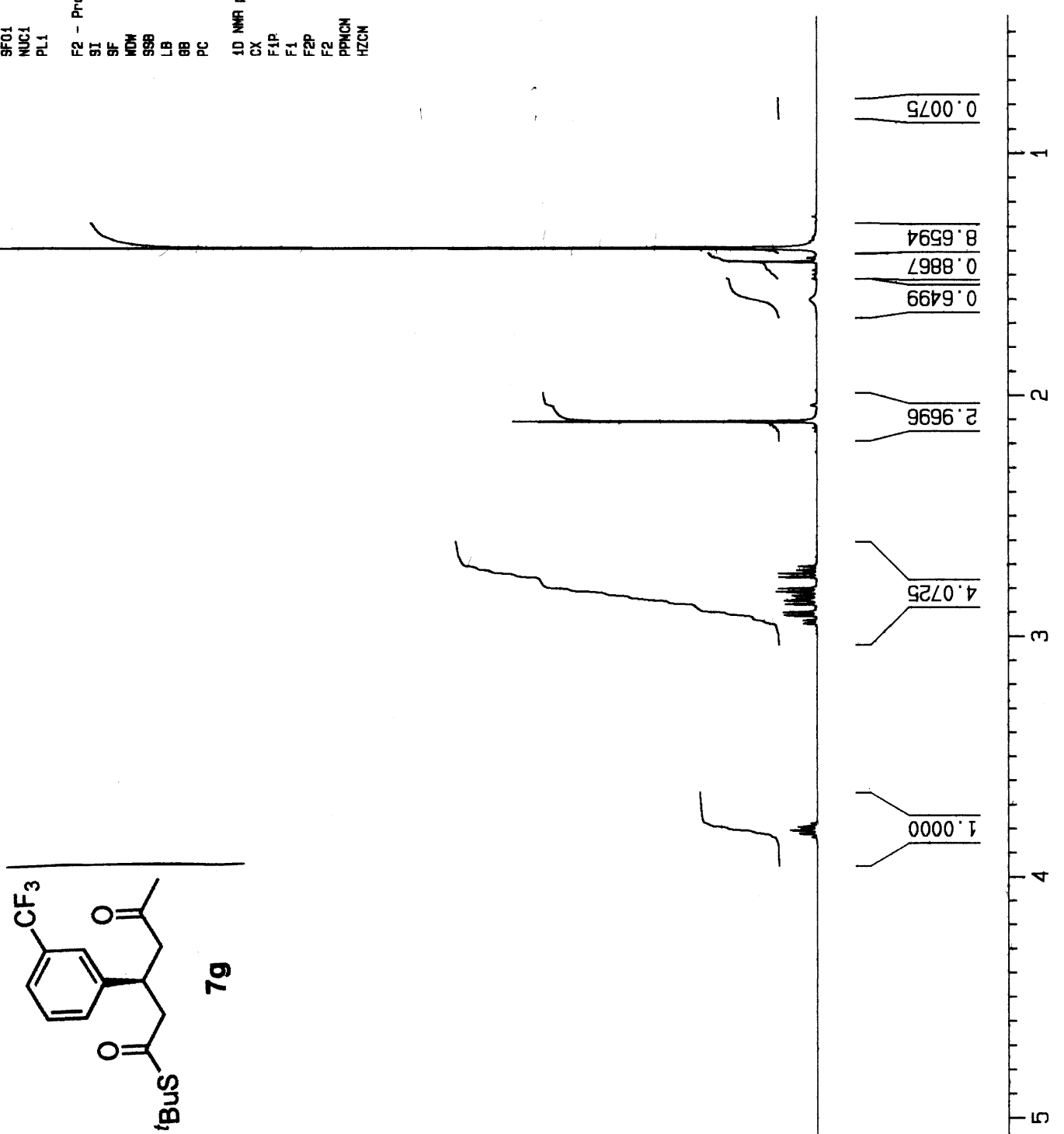

टLI'G
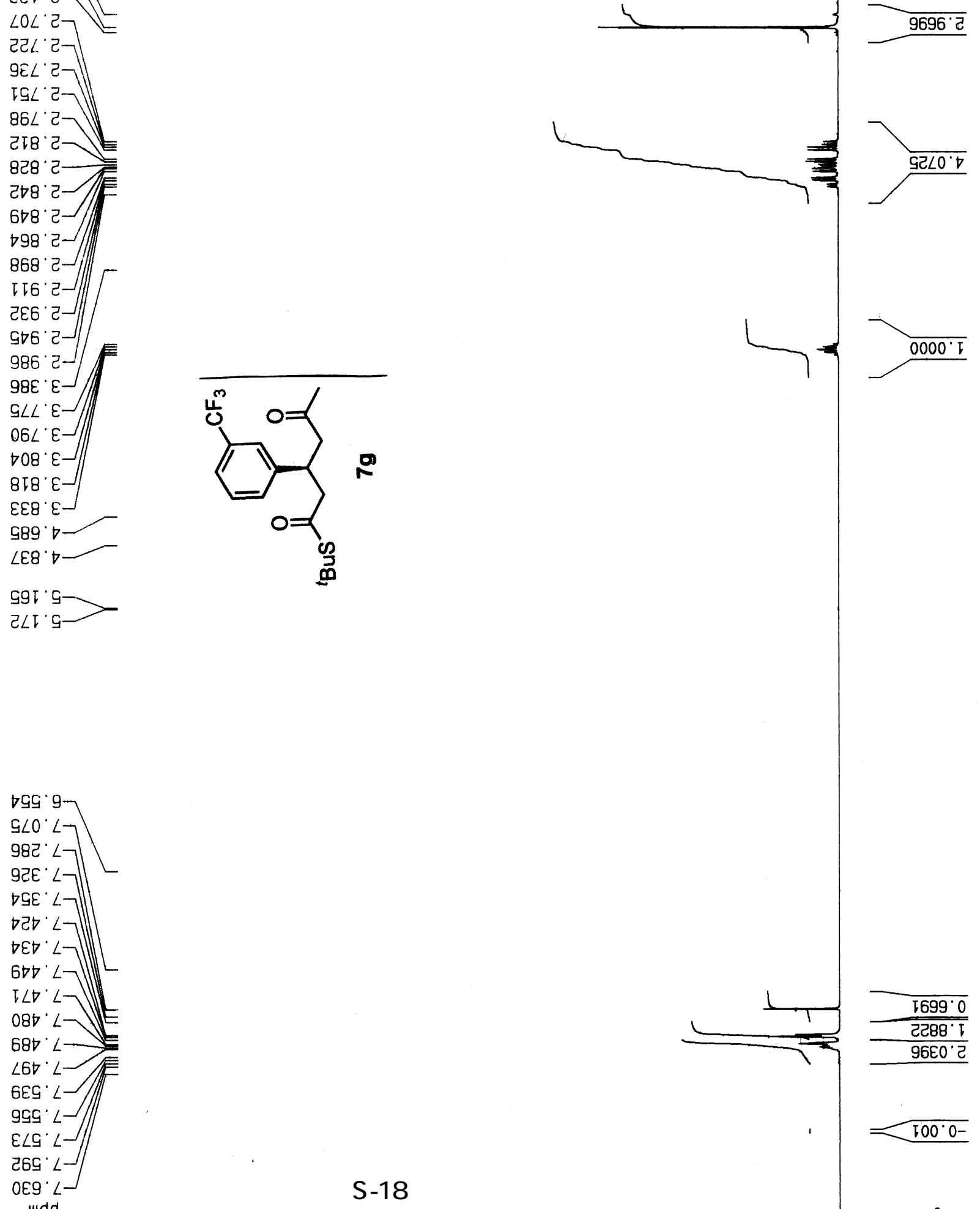


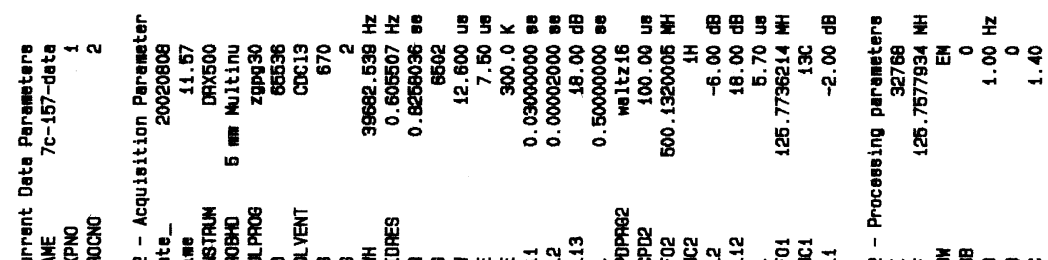

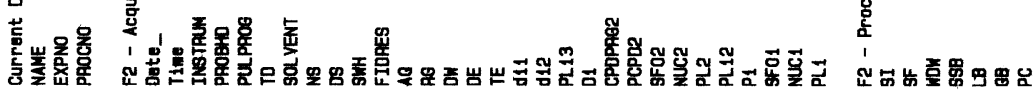
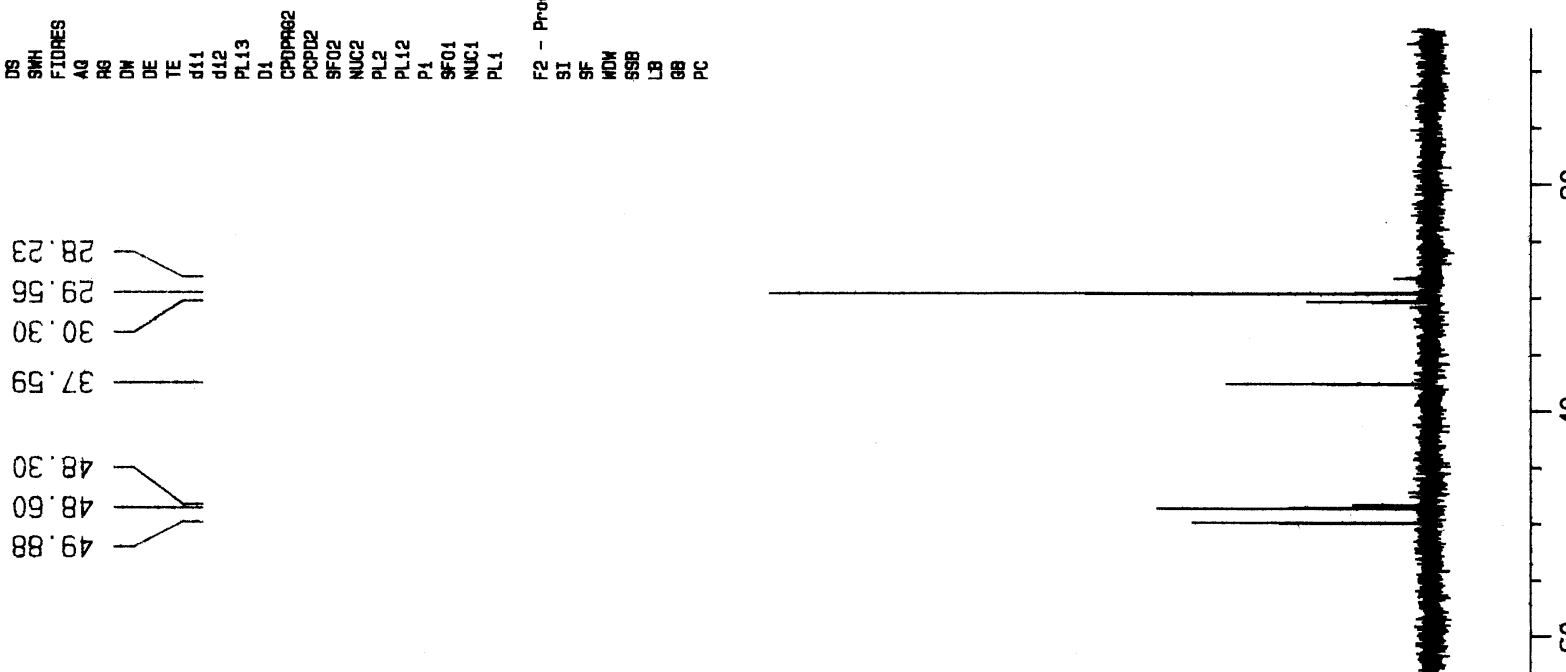

$i \angle \cdot 9 L$

$\angle 6^{\circ} 9 L$

टट ' $\angle L$

$\angle 9^{\circ} \varepsilon 己$

$0 L \varepsilon ट T$

GT'Dटा

EI GटT-

$96.82 \tau$

$90^{\circ}$ TET

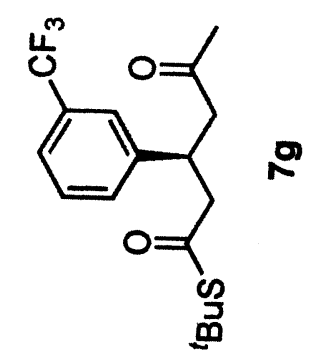

$99 \cdot \varepsilon \triangleright I-$

$\angle 0^{\circ} 861$

$58^{\circ} .902$ 

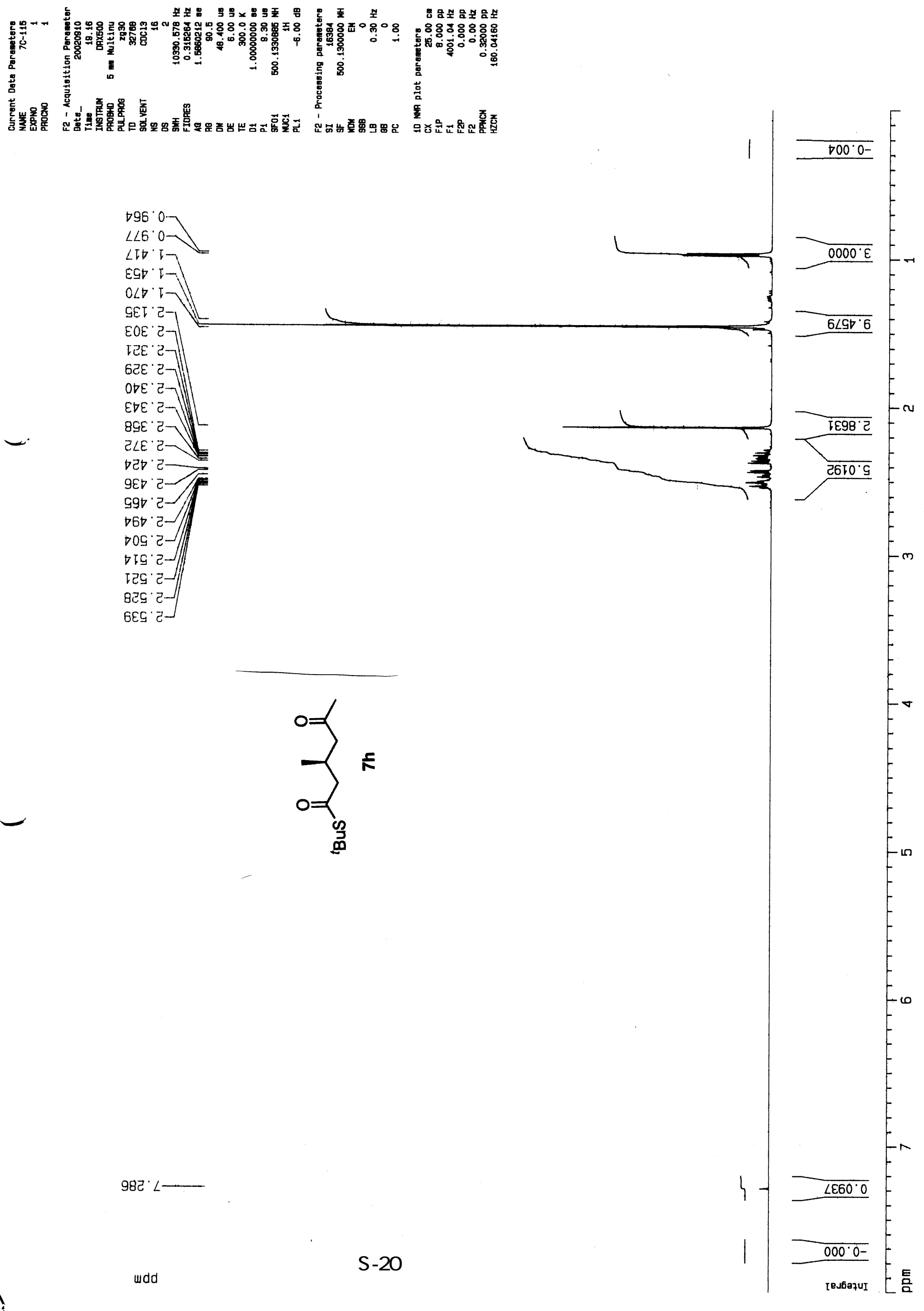

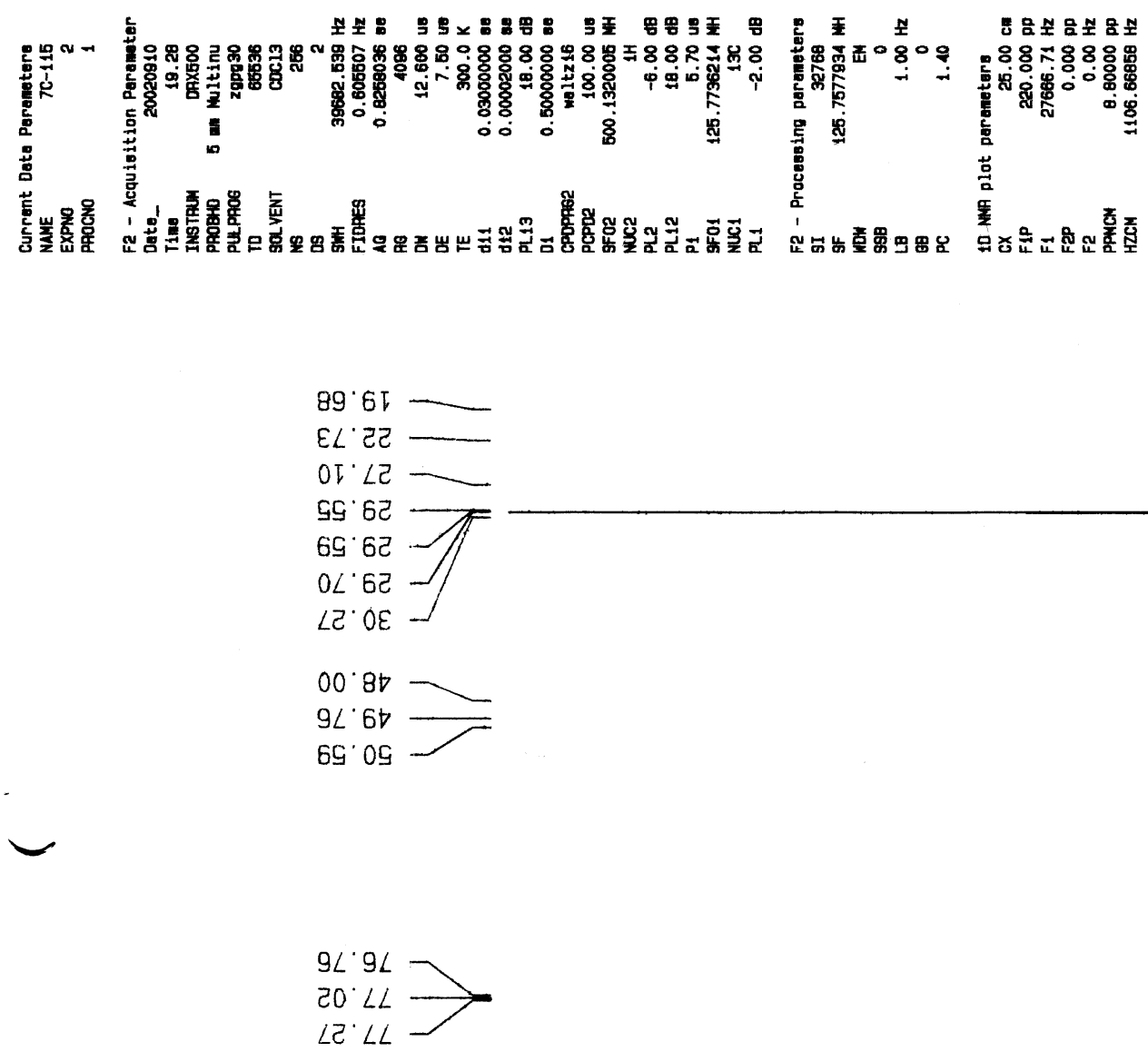

.




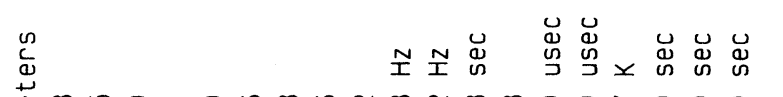

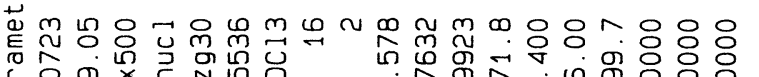

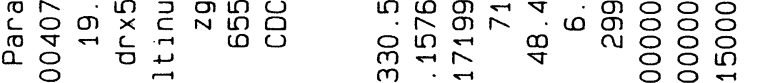

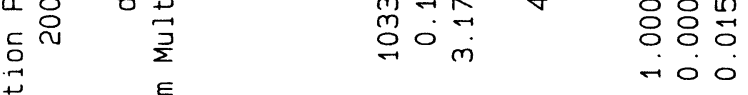

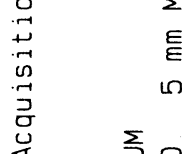

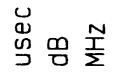

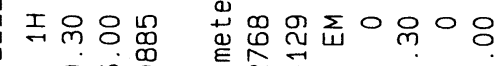

폰

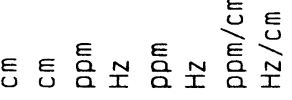
응으궁ㅇㅇㅇㅁㅇ

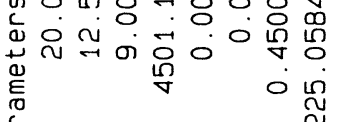
임 $\stackrel{\mathrm{o}}{\mathrm{a}}$

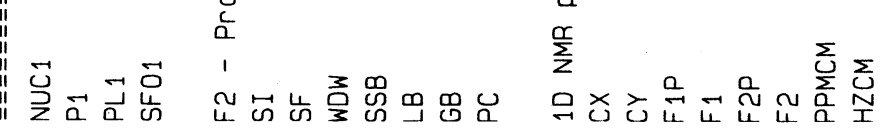

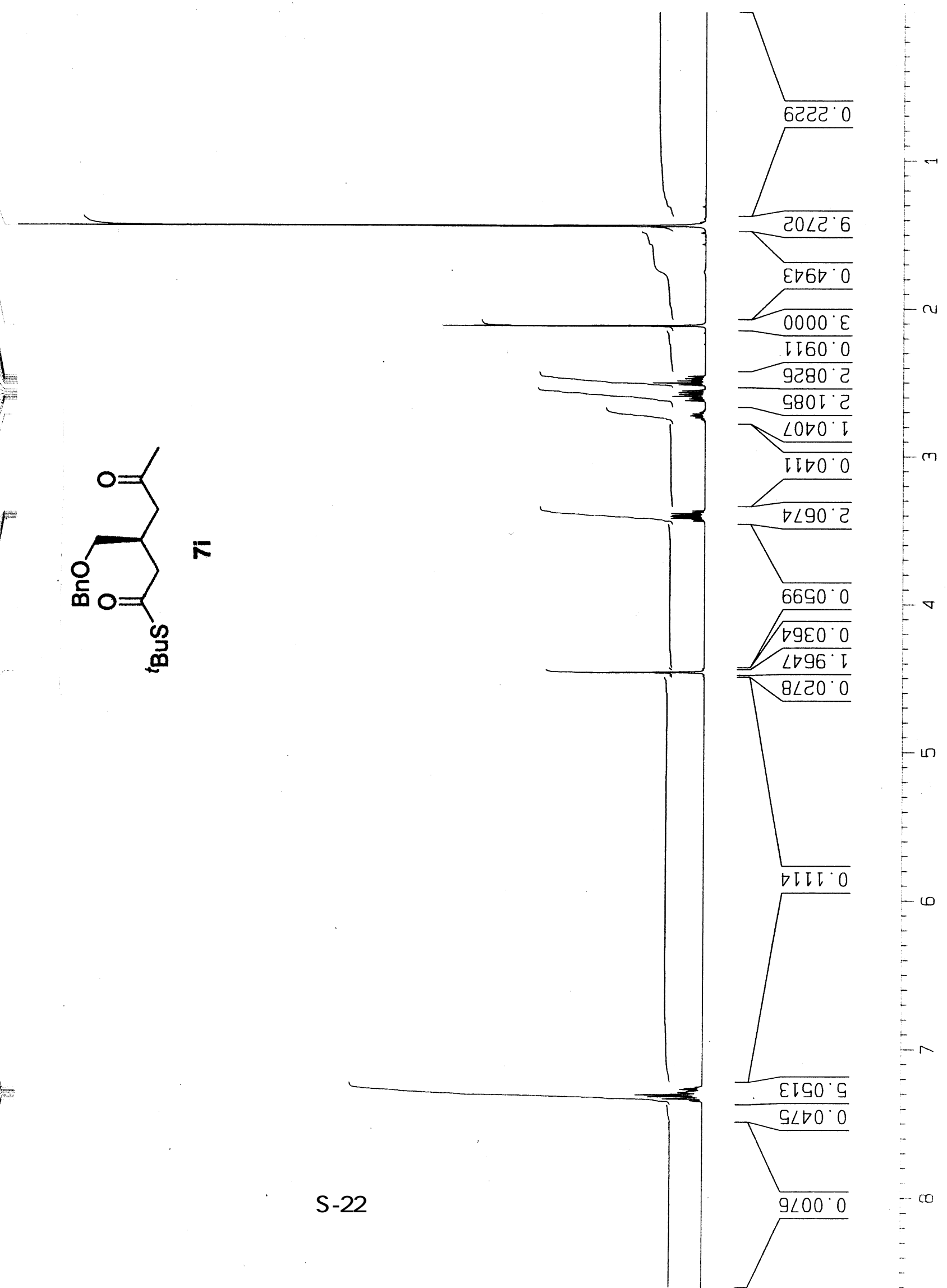




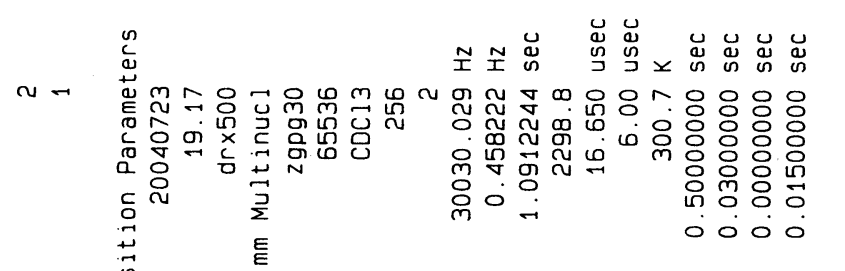

岗

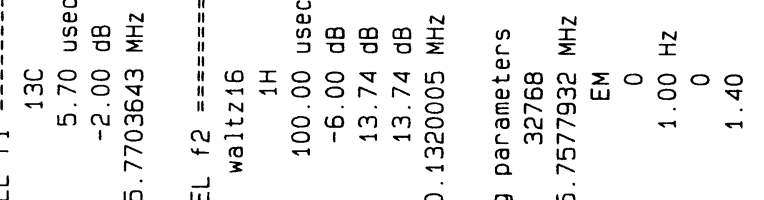

튼

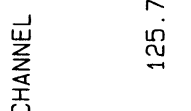

哩

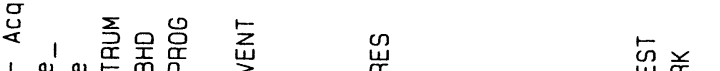

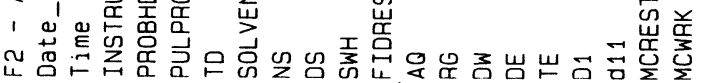

$\overrightarrow{\mathrm{S}}-\overrightarrow{\mathrm{O}}$

宫

ช

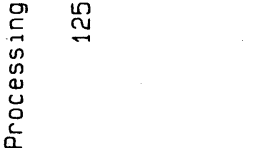

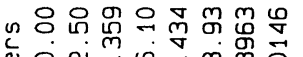

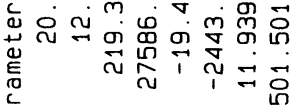

骂

蒙

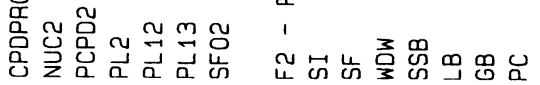

产

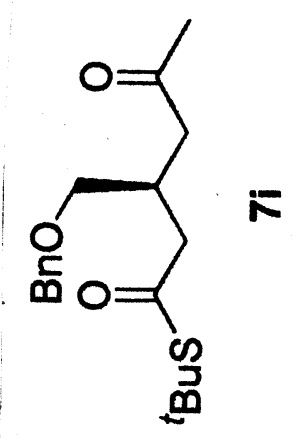



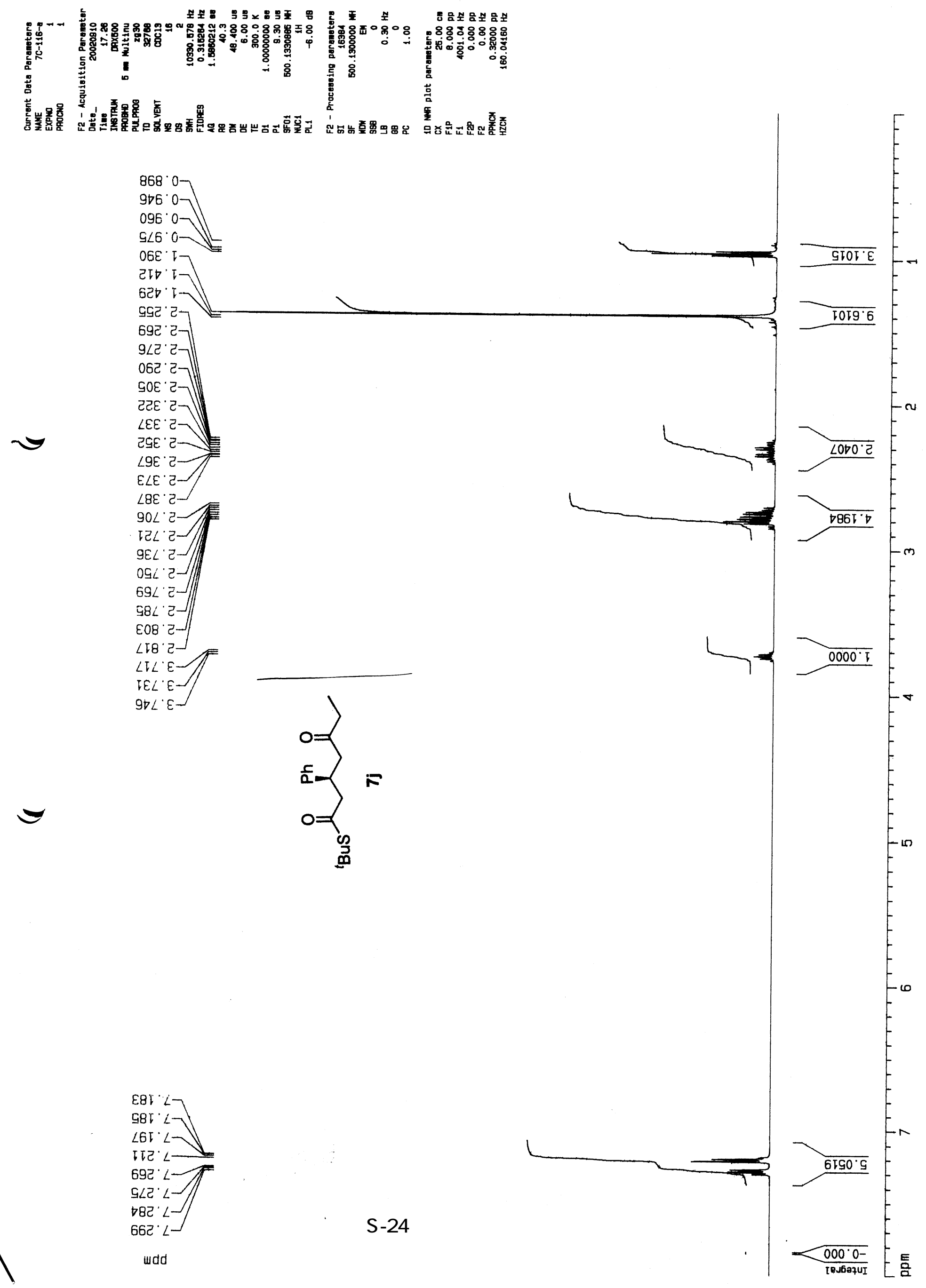

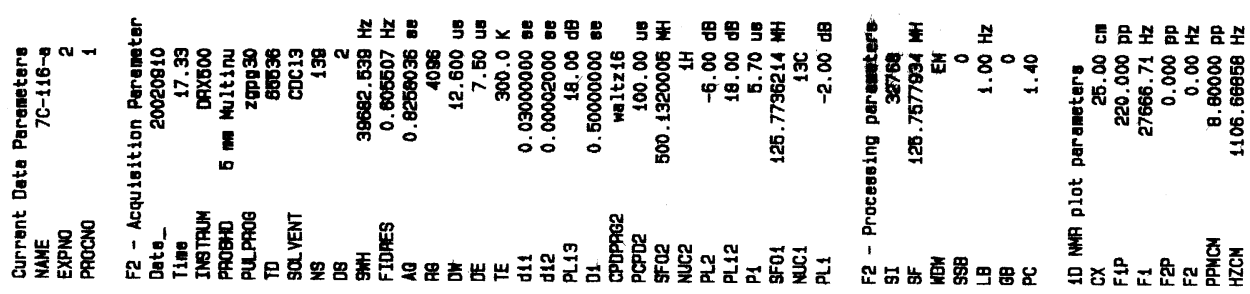
टG' $L$

ฮाจ

ع9. टट

89.62

$8 \varepsilon^{\circ} 9 \varepsilon$

$\varepsilon O^{\circ} 8 E$

$08^{\circ} \angle D$

$\nabla 0^{\circ} 8 \mathrm{~V}$

$9 E^{\circ} O S$

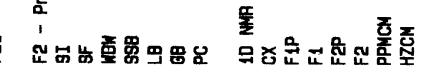

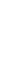

$18.9 L$

$\angle O^{\circ} \angle L$

टE $\angle L \longrightarrow$

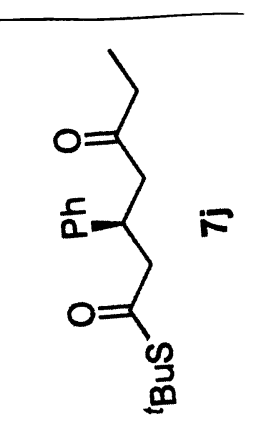

$\varepsilon L \cdot 921$

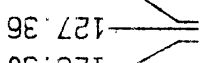

OS.8टT-

GL'己ヤI-
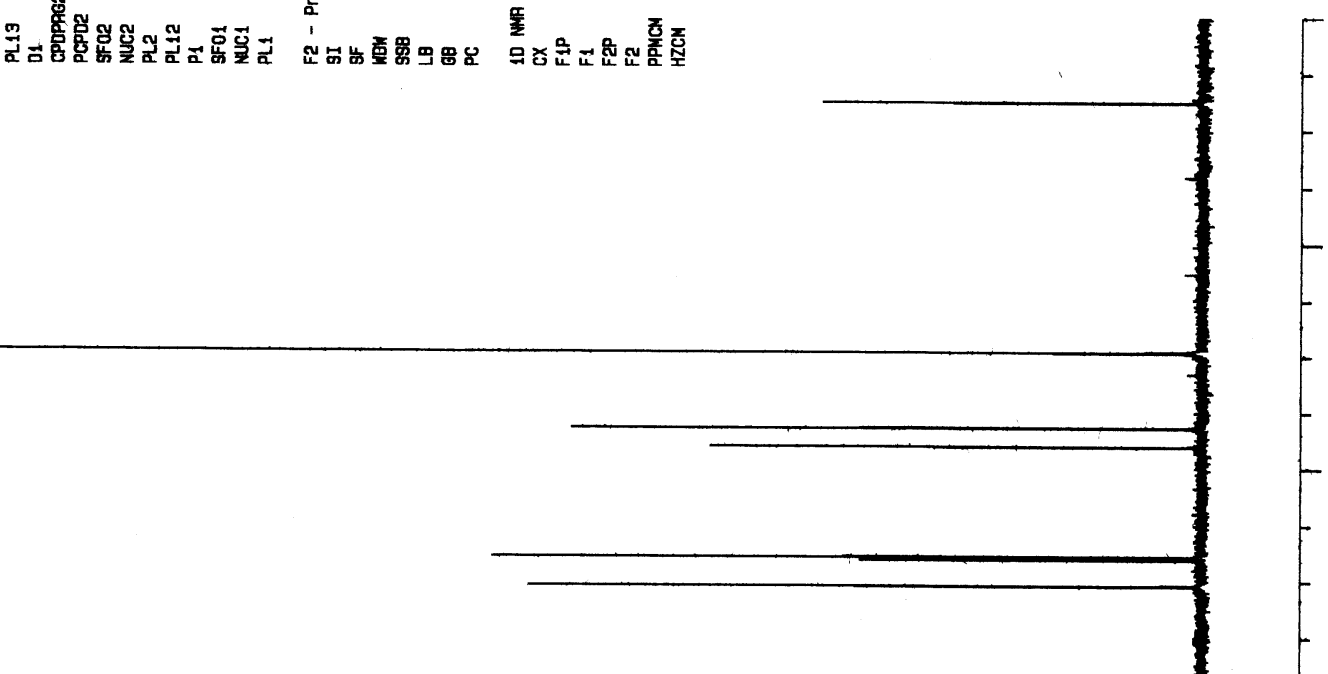


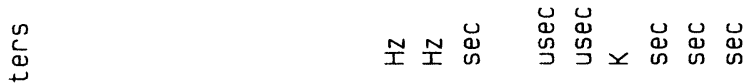

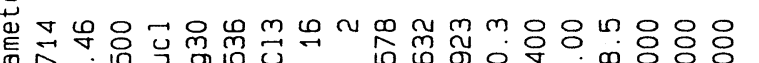

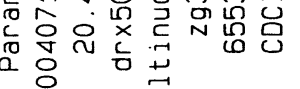

동

동

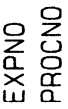

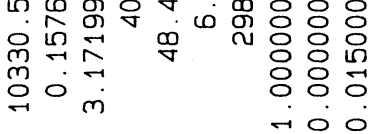

崫

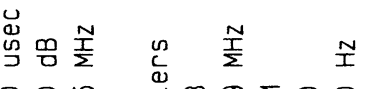

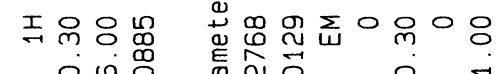

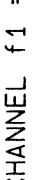

$\stackrel{0}{\circ} 1 \stackrel{\mathrm{m}}{\mathrm{m}}$

0
6
0
0
0
0
5
0
5
0
0
0
0

응
용

$\vec{y} \rightarrow \overrightarrow{0} 1 \quad$ 紊

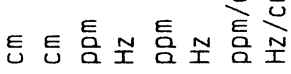

응ㅇㅇ응ㅇㅇㅇㅇㅇㅁㅇ

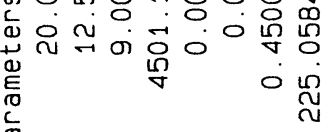

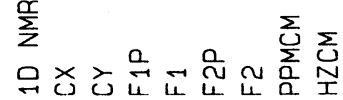
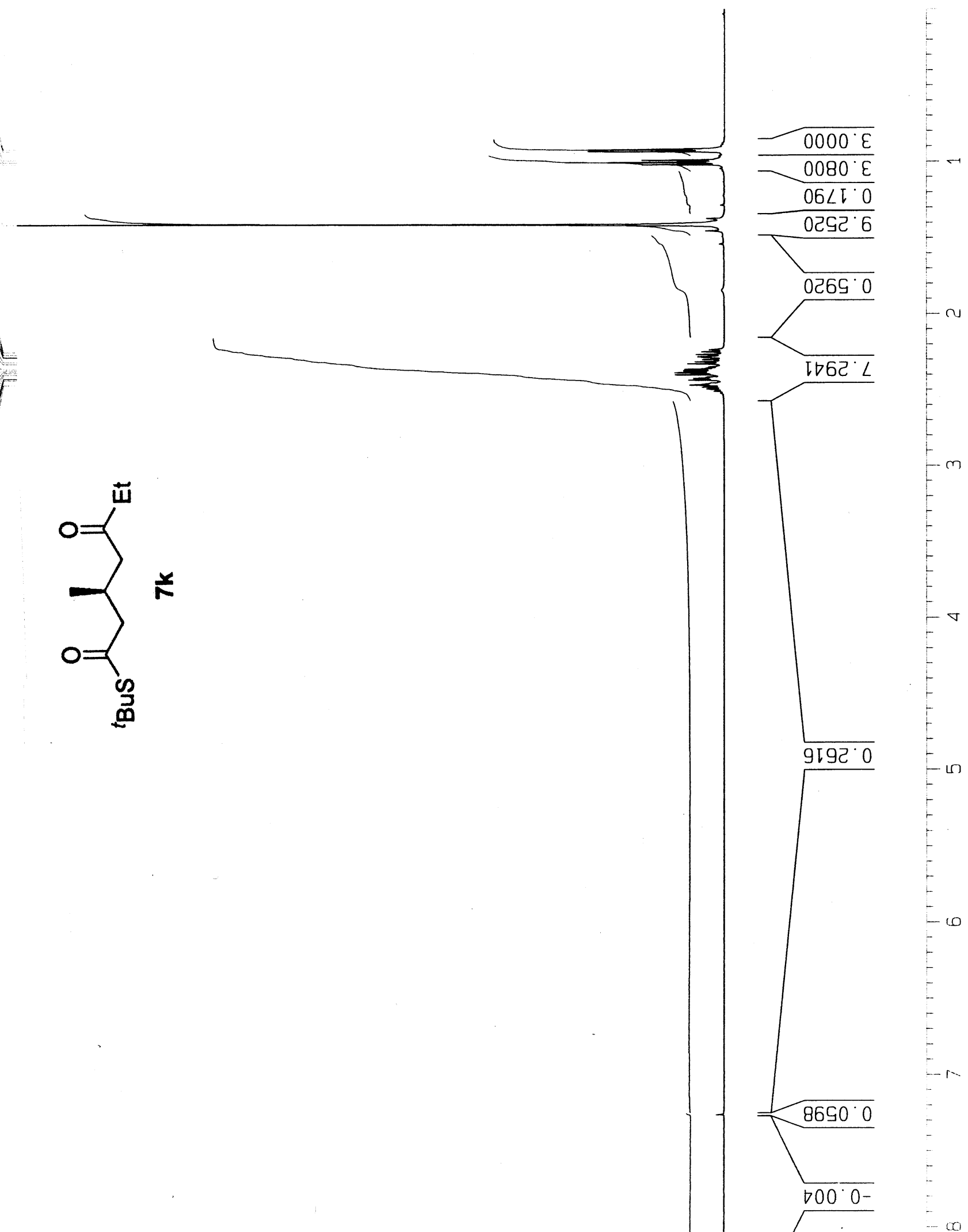


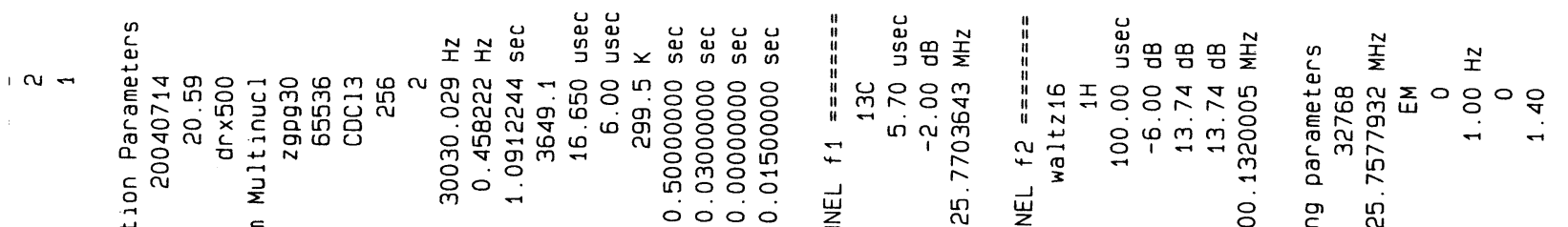

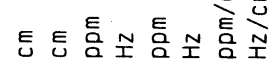

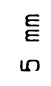

嵌 崩

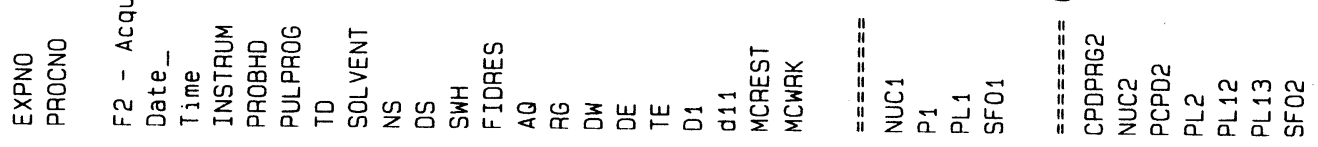
状客

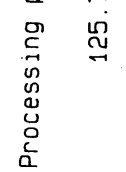

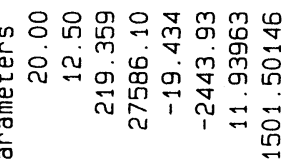

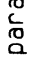
蒙

岀出出预界田思员

变

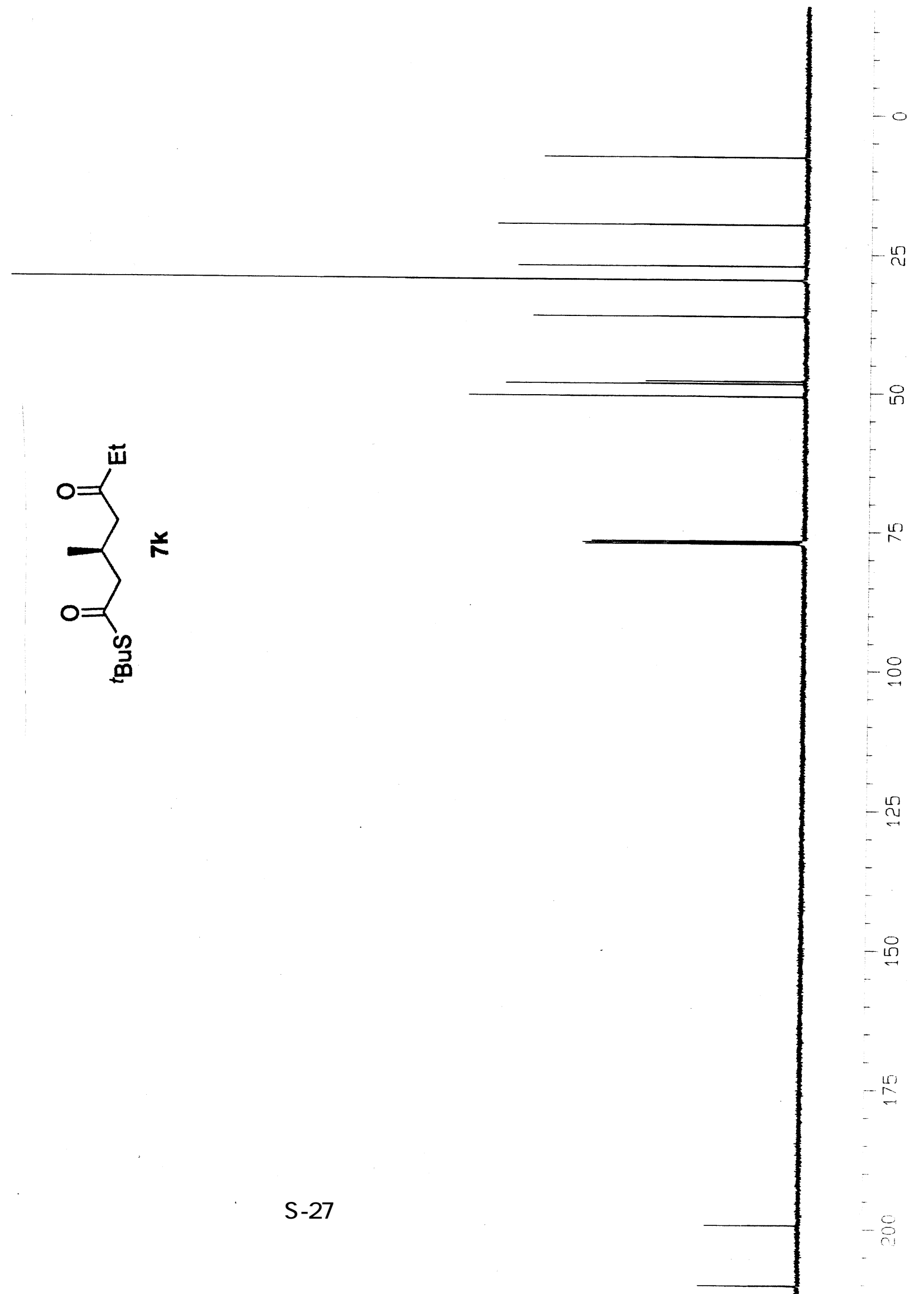




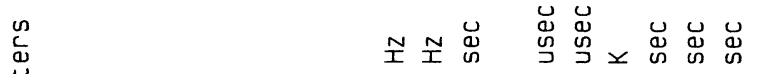

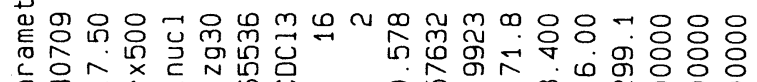

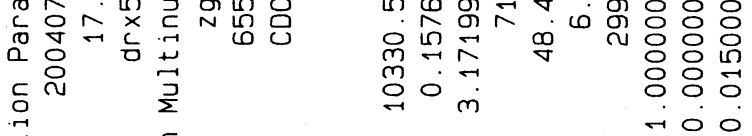

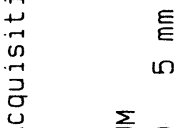
율은

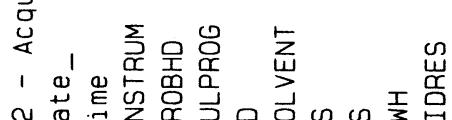
-i०

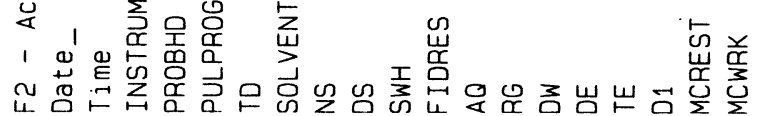

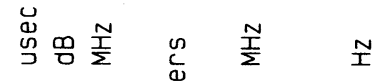

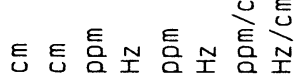

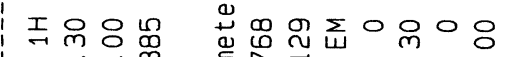
폰 $\|$

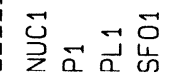

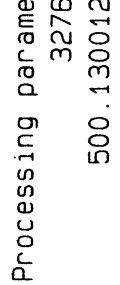

$1, \frac{\alpha}{\sum_{2}}$

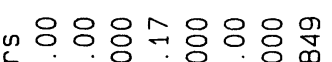

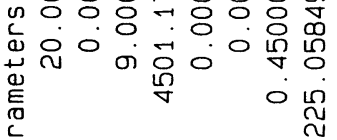
高 蒙

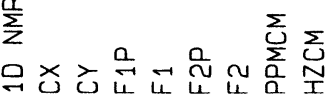

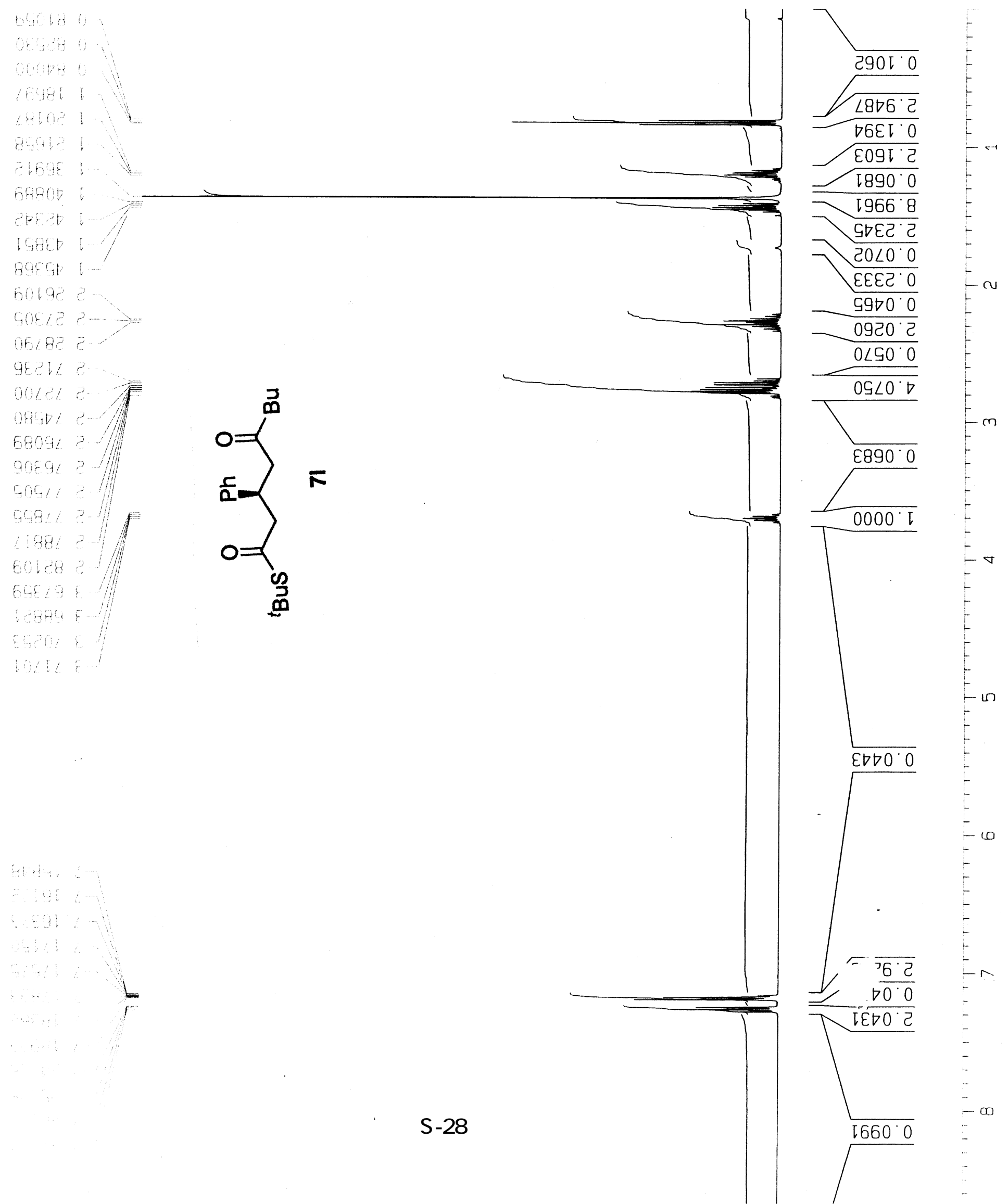




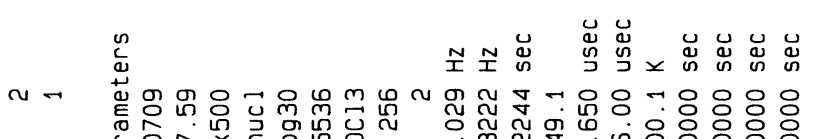

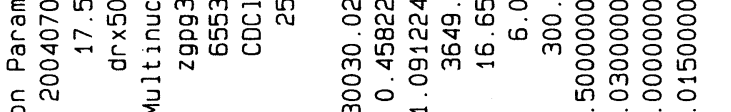

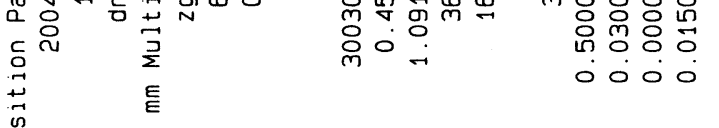

궁
แ

悹
苑 늑요욤

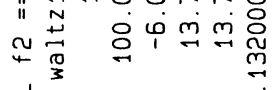
崖

ก

空

"I "
|

嵌

"
号

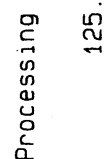

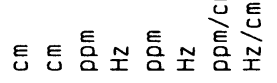

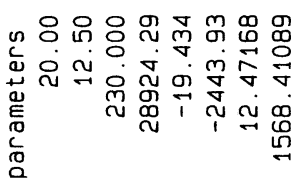

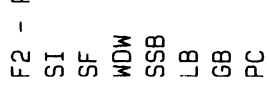

蒙

兴

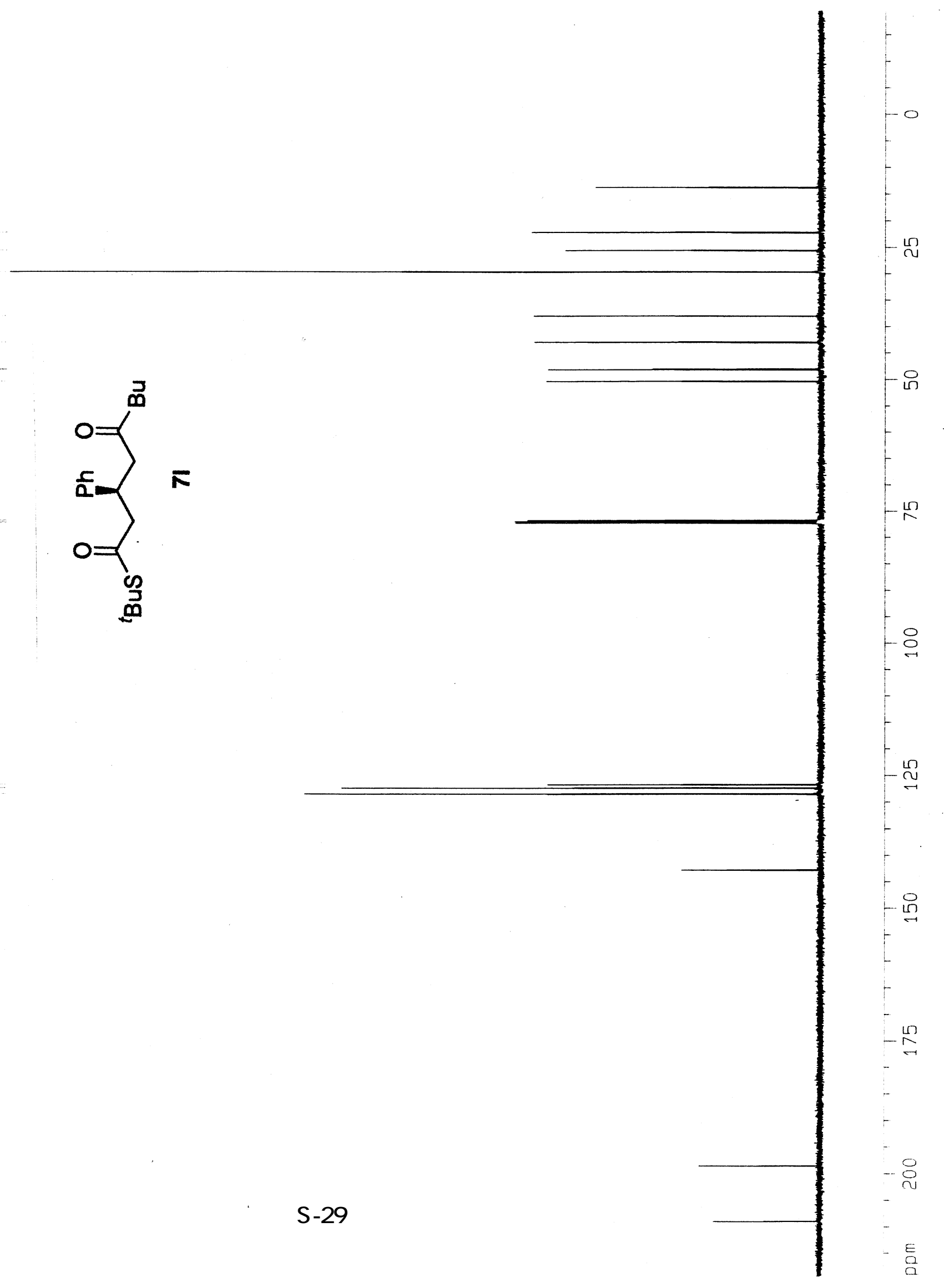



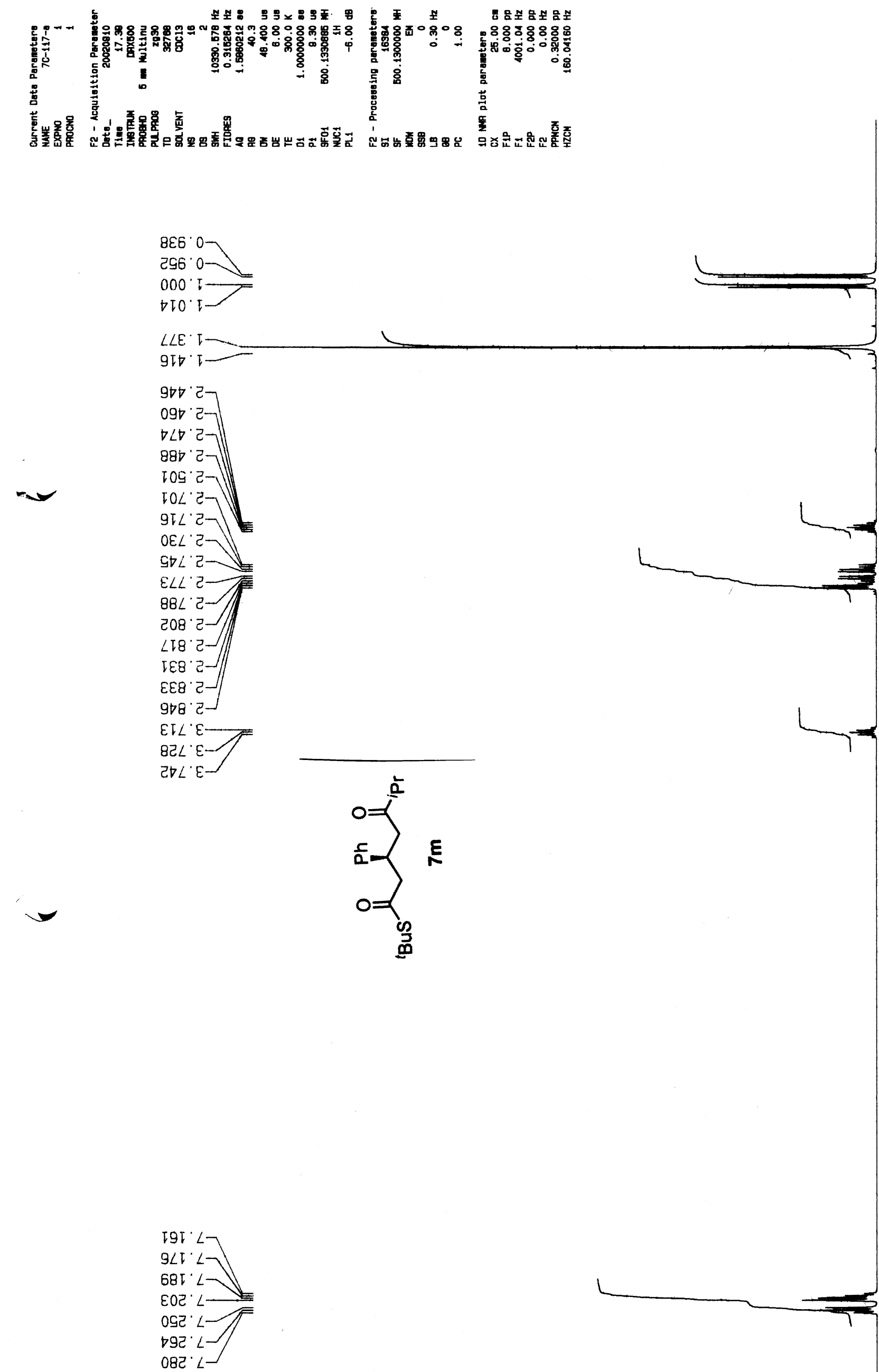


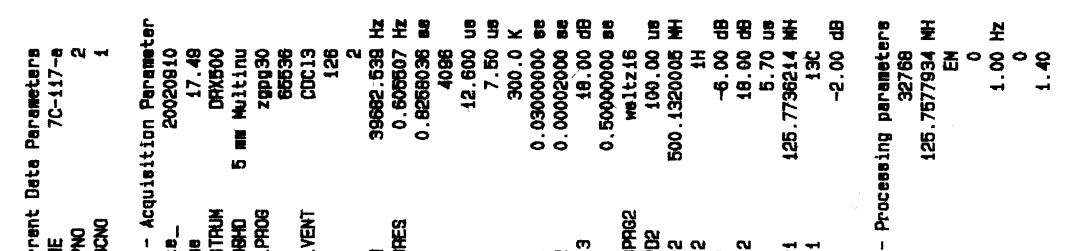

훙을소믐소 몸소

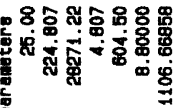

莒

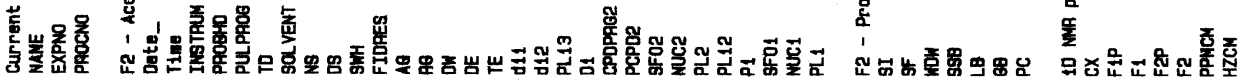

$98^{\circ} \angle \tau$

$\nabla 9 \cdot 62$

$\angle 8^{\circ} \angle E^{\circ}$

$20^{\circ}$ it

$16^{\circ} 95$

$86^{\circ} \angle \nabla$

6I.09

$98 \cdot 9 L$

टT' $L L$

$\angle E \cdot L L$
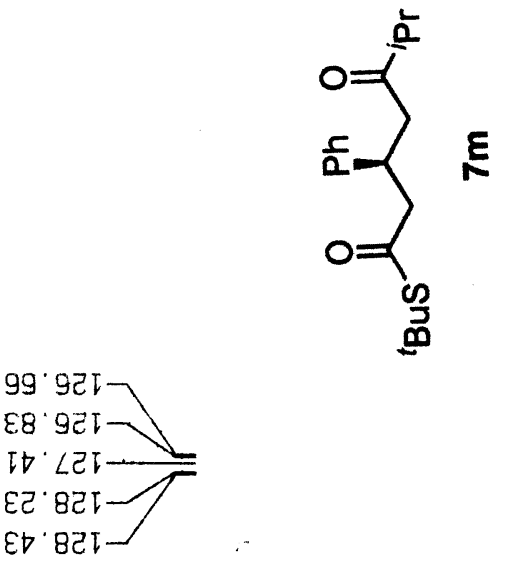

$06^{\circ}$ टD厂

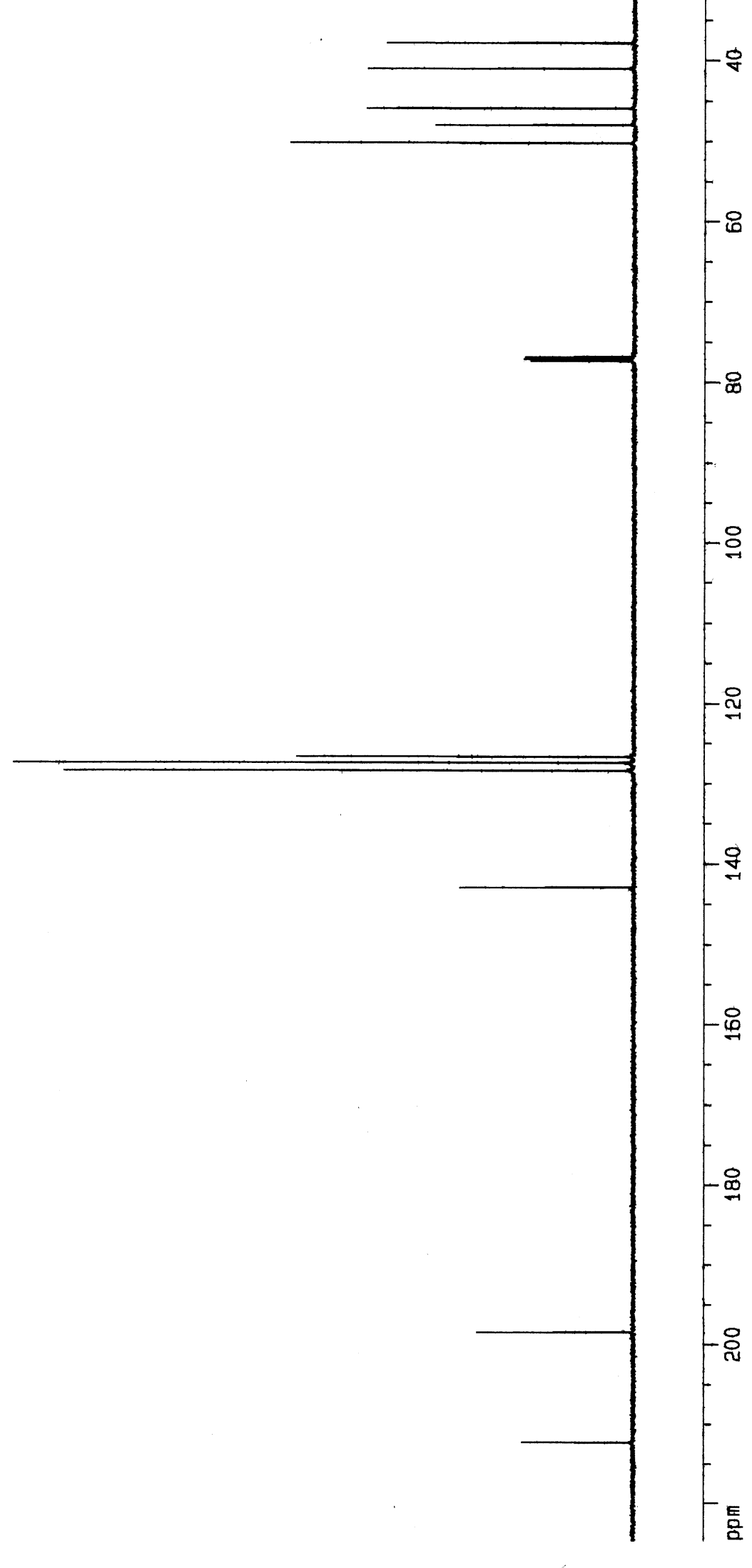

$80^{\circ} 86 \tau$ 